\title{
Meridional destabilizing number of knots
}

\author{
TOSHIO SAITO
}

\begin{abstract}
We define the meridional destabilizing number of a knot. This together with Heegaard genus (or tunnel number) gives a binary complexity of knots. We study its behavior under connected sum of tunnel number one knots.
\end{abstract}

$57 \mathrm{M} 25 ; 57 \mathrm{~N} 10$

\section{Introduction}

\subsection{Backgrounds}

From a viewpoint of Heegaard theory, we have two types of natural positions of knots in connected closed orientable 3-manifolds: (i) a bridge position with respect to a Heegaard surface, and (ii) a core position of a handlebody bounded by a Heegaard surface. A Heegaard surface of type (ii) corresponds to that of a knot exterior. Hence it has a close connection to Heegaard genus and tunnel number of knots defined below.

Let $M$ be a connected closed orientable 3-manifold and $\left(V_{1}, V_{2} ; S\right)$ a (genus $g$ ) Heegaard splitting of $M$, that is, (1) $V_{1}$ and $V_{2}$ are (genus $g$ ) handlebodies, (2) $V_{1} \cup V_{2}=M$ and (3) $V_{1} \cap V_{2}=\partial V_{1}=\partial V_{2}=S$. Such a surface $S$ is called a Heegaard surface of $M$. A knot $K$, that is, a connected closed 1 -manifold in $M$ is in a $(g, b)$-bridge position if $K$ is in a $b$-bridge position with respect to a Heegaard surface of genus $g$ (see Section 2.1 for the precise definition). Set $\mathcal{M}=(M, K)$, $\mathcal{V}_{i}=\left(V_{i}, V_{i} \cap K\right)(i=1,2)$ and $\mathcal{S}=(S, S \cap K)$. If a Heegaard splitting $\left(V_{1}, V_{2} ; S\right)$ of $M$ gives a $(g, b)$-bridge position of $K$, then $\left(\mathcal{V}_{1}, \mathcal{V}_{2} ; \mathcal{S}\right)$ is called a $(g, b)$-bridge splitting of $\mathcal{M}$, and $\mathcal{S}$ is called a $(g, b)$-bridge surface. This is introduced by Doll [2] and is a natural generalization of classical bridge decompositions of knots in the 3-sphere $S^{3}$.

A Heegaard splitting $\left(V_{1}, V_{2} ; S\right)$ of $M$ is also called a Heegaard splitting of $\mathcal{M}=$ $(M, K)$ if $K \subset V_{i}$, say $i=1$, and the exterior of $K$ in $V_{1}$ is a compression body. Such a surface $S$ is also called a Heegaard surface of $\mathcal{M}$. The Heegaard genus of $K \subset M$, denoted by $\operatorname{hg}(K)$, is the minimal value $g$ such that $\mathcal{M}$ admits a Heegaard surface of genus $g$. We notice that $t(K):=\mathrm{hg}(K)-1$ is called the tunnel number of $K \subset M$. 
Let $\left(V_{1}, V_{2} ; S\right)$ be a genus $g$ Heegaard splitting of $\mathcal{M}=(M, K)$ with $K \subset V_{1}$. Suppose that there are compressing disks $D_{i}(i=1,2)$ of $V_{i}$ such that $D_{1}$ intersects $K$ transversely in a single point and that $\partial D_{1}$ intersects $\partial D_{2}$ transversely in a single point. Then $S$ is said to be meridionally stabilized. Under this condition we obtain a $(g-1,1)$-bridge splitting of $\mathcal{M}$ as follows. Let $V_{1}^{\prime}$ be a 3 -manifold obtained by cutting $V_{1}$ along $D_{1}$. Since $D_{1}$ is non-separating in $V_{1}$, we see that $V_{1}^{\prime}$ is a handlebody of genus $g-1$. Moreover, $V_{1}^{\prime} \cap K$ is a trivial arc in $V_{1}^{\prime}$, that is, $V_{1}^{\prime} \cap K$ is a simple arc which is isotopic into $\partial V_{1}^{\prime}$ relative to boundary. Attaching a (closed) regular neighborhood of $D_{1}$ in $V_{1}$ to $V_{2}$, we obtain a 3-manifold $V_{2}^{\prime}$ which is also a handlebody of genus $g-1$. We also see that $V_{2}^{\prime} \cap K$ is a trivial arc in $V_{2}^{\prime}$. Therefore $S^{\prime}:=\partial V_{1}^{\prime}=\partial V_{2}^{\prime}$ gives a $(g-1,1)$-bridge position of $K$, that is, $\left(\mathcal{V}_{1}^{\prime}, \mathcal{V}_{2}^{\prime} ; \mathcal{S}^{\prime}\right)$ is a $(g-1,1)$-bridge splitting of $\mathcal{M}$, where $\mathcal{V}_{i}^{\prime}=\left(V_{i}^{\prime}, V_{i}^{\prime} \cap K\right)(i=1,2)$ and $\mathcal{S}^{\prime}=\left(S^{\prime}, S^{\prime} \cap K\right)$. We call this operation meridional destabilization. We can similarly define a meridionally stabilized $(g, b)$-bridge surface and obtain a $(g-1, b+1)$-bridge surface from such a surface by meridional destabilization.

It could not be said that there is a close relationship between a bridge number $b_{g}(K)$ and Heegaard genus $\operatorname{hg}(K)$, where $b_{g}(K)$ is the minimal bridge number of $K$ with respect to a genus $g$ Heegaard surface of $M$. If, of course, $\mathcal{M}$ admits a $(g, b)$-bridge position, then we obtain a genus $g+b$ Heegaard splitting of $\mathcal{M}$ by repeating the converse operation of meridional destabilization and hence we see $\operatorname{hg}(K) \leq g+b$. However, Minsky, Moriah and Schleimer [10, Theorem 4.2] showed that for any integers $g \geq 2$ and $b \geq 1$, there is a knot $K \subset S^{3}$ with $\operatorname{hg}(K)=g$ such that $K$ does not admit a $(g, b)$-bridge position (see also Johnson and Thompson [5] for the case of $g=2$ ). In this paper, we define meridional destabilizing number of a knot $K \subset M$ as follows:

Definition 1.1 Let $K$ be a knot in a connected closed orientable 3-manifold $M$. Meridional destabilizing number of $K$, denoted by $\operatorname{md}(K)$, is defined by the maximal number of $m$ such that $\mathcal{M}=(M, K)$ admits a $(\mathrm{hg}(K)-m, m)$-bridge position. In particular, $\operatorname{md}(K)=0$ if none of the minimal genus Heegaard splittings of $\mathcal{M}$ are meridionally stabilized.

By the definition above, we see that $\operatorname{md}(K) \leq \operatorname{hg}(K)$ for any knot $K$.

Notation 1.2 Let $K$ be a knot in $S^{3}$. We describe $K \in \mathcal{K}_{g}^{m}$ if $\operatorname{hg}(K)=g$ and $\operatorname{md}(K)=m$.

For example, $K \in \mathcal{K}_{1}^{1}$ if and only if $K$ is a trivial knot. We can divide tunnel number one knots into three families, $\mathcal{K}_{2}^{2}, \mathcal{K}_{2}^{1}$ and $\mathcal{K}_{2}^{0}$. Knots in $\mathcal{K}_{2}^{2}$ are non-trivial 2-bridge knots, those in $\mathcal{K}_{2}^{1}$ are $(1,1)$-knots which are not 2 -bridge knots, and those in $\mathcal{K}_{2}^{0}$ are the other tunnel number one knots. 


\subsection{Results}

We study behavior of meridional destabilizing number under connected sum of knots. Let $K$ be a knot in $S^{3}$. We denote by $n K$ the connected sum of $n$ copies of $K$. Then we have: $n \leq t(n K) \leq n \cdot t(K)+(n-1)$ or equivalently

$$
n+1 \leq \operatorname{hg}(n K) \leq n \cdot \operatorname{hg}(K) .
$$

The upper bound is well-known and is easy to understand. However, the lower bound is highly non-trivial and is obtained by Scharlemann and Schultens [15, Theorem 14]. If $\operatorname{md}(K) \neq 0$, then we also have $\operatorname{hg}(n K) \leq n \cdot \operatorname{hg}(K)-n+1$ and $\operatorname{md}(n K) \neq 0$ (see Proposition 2.14). Hence if $\operatorname{hg}(K)=2$ and $\operatorname{md}(K) \neq 0$, then $\operatorname{hg}(n K)=n+1$. It follows from Schubert's formula on bridge number [16] that $K$ is a 2-bridge knot if and only if $n K$ is an $(n+1)$-bridge knot. Similarly we have:

Observation 1.3 $K_{1}, \ldots, K_{n} \in \mathcal{K}_{2}^{2}$ if and only if $K_{1} \# \cdots \# K_{n} \in \mathcal{K}_{n+1}^{n+1}$.

In this paper, we show:

Theorem 1.4 Let $K$ be a knot in $S^{3}$.

(1) If $K_{i} \in \mathcal{K}_{2}^{1}(i=1,2,3)$, then $K_{1} \# K_{2} \in \mathcal{K}_{3}^{1}$ and $K_{1} \# K_{2} \# K_{3} \in \mathcal{K}_{4}^{1}$.

(2) If $K_{j} \in \mathcal{K}_{2}^{0}(j=1,2)$, then $K_{1} \# K_{2} \in \mathcal{K}_{4}^{0}$ or $\mathcal{K}_{4}^{1}$.

The most famous examples of knots in $\mathcal{K}_{2}^{0}$ would be so-called MSY knots $K_{\text {MSY }}$ introduced by Morimoto, Sakuma and Yokota [14]. It follows from Morimoto [11, Corollary 2] that $\operatorname{hg}\left(2 K_{\mathrm{MSY}}\right)=4$. Since $K_{\mathrm{MSY}}$ admits a $(1,2)$-bridge position, we see that $\operatorname{md}\left(2 K_{\mathrm{MSY}}\right) \geq 1$ (see Kobayashi and Rieck [6, Theorem A.1], see also Proposition 2.14). Therefore we have the following as a corollary of Theorem 1.4.

Corollary 1.5 $K_{\mathrm{MSY}} \in \mathcal{K}_{2}^{0}$ and $2 K_{\mathrm{MSY}} \in \mathcal{K}_{4}^{1}$.

On the other hand, Kobayashi and Rieck [8] showed that there is a knot $K \in \mathcal{K}_{2}^{0}$ with $2 K \in \mathcal{K}_{4}^{0}$. This implies that (2) of Theorem 1.4 is best possible. As a summary, we have Figure 1.

Remark 1.6 More generally, Kobayashi and Rieck proved the following: given an integer $m \geq 1$, there are infinitely many knots $K$ in $S^{3}$ such that $\operatorname{hg}\left(m^{\prime} K\right)=m^{\prime} \cdot \operatorname{hg}(K)$ for any positive integer $m^{\prime} \leq m$ (see Kobayashi and Rieck [7, Corollary 1.6]). This implies that given an integer $n \geq 1$, there are infinitely many knots $K \in \mathcal{K}_{2}^{0}$ such that $\operatorname{md}\left(n^{\prime} K\right)=0$ for any positive integer $n^{\prime} \leq n$. 


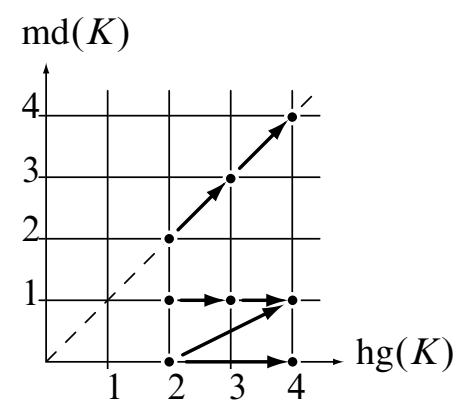

Figure 1: Relation between $\mathrm{hg}(\cdot)$ and $\operatorname{md}(\cdot)$ under connected sum

Based on the results above, we would like to ask some questions on tunnel number one knots.

Question 1.7 (1) Is there a knot $K \in \mathcal{K}_{2}^{1}$ with $\operatorname{md}(n K)>1$ for some integer $n$ ?

(2) Is there a knot $K \in \mathcal{K}_{2}^{0}$ with $\operatorname{md}(n K)=0$ for any integer $n$ ?

It would be much interesting and challenging to take the connected sum of knots with tunnel number greater than one, because there is a possibility of sub-additivity of tunnel number under connected sum (see Kobayashi and Saito [9, Assertion 6.4]).

\section{Preliminaries}

Throughout this paper, we work in the piecewise linear category. Let $B$ be a submanifold of a manifold $A$. The notation $\eta(B ; A)$ denotes a (closed) regular neighborhood of $B$ in $A$. By $\operatorname{Ext}(B ; A)$, we mean the exterior of $B$ in $A$, that is, $\operatorname{Ext}(B ; A)=\operatorname{cl}(A \backslash \eta(B ; A))$, where $\operatorname{cl}(\cdot)$ means the closure. The notation $|\cdot|$ indicates the number of connected components. Let $M$ be a connected compact orientable 3-manifold. A link in $M$ is a closed 1-manifold in $M$ and a knot in $M$ is a connected closed 1-manifold in $M$. Let $J$ be a 1 -manifold properly embedded in $M$ and $F$ a surface properly embedded in $M$. Here, a surface means a connected compact 2 -manifold. We always assume that $J$ is not split, that is, there is no 2 -sphere in $M \backslash J$ which separates the components of $J$, and also assume that $F$ intersects $J$ transversely. Set $\mathcal{M}=(M, J)$ and $\mathcal{F}=(F, F \cap J)$. For convenience, we also call $\mathcal{F}$ a surface, and we say that $\mathcal{F}$ is closed if $F$ is closed. Whenever we use such calligraphic symbols, we consider not only a $2-$ or 3 -manifold but intersections with a 1-manifold. 


\subsection{Fundamental definitions}

A simple closed curve or a simple arc properly embedded in $F \backslash J$ is said to be trivial in $\mathcal{F}$ if it cuts off a disk from $F$ which is disjoint from $J$. A simple closed curve properly embedded in $F \backslash J$ is said to be inessential in $\mathcal{F}$ if it bounds a disk in $F$ intersecting $J$ in at most one point. A simple closed curve properly embedded in $F \backslash J$ is said to be essential in $\mathcal{F}$ if it is not inessential in $\mathcal{F}$. A surface $\mathcal{F}$ is compressible in $\mathcal{M}$ if there is a disk $D \subset M \backslash J$ such that $D \cap F=\partial D$ and $\partial D$ is essential in $\mathcal{F}$. Such a disk $D$ is called a compressing disk of $\mathcal{F}$. We say that $\mathcal{F}$ is incompressible in $\mathcal{M}$ if $\mathcal{F}$ is not compressible in $\mathcal{M}$.

Suppose that $\partial M \neq \varnothing$ and $\partial F \neq \varnothing$. We say that $\mathcal{F}$ is $\partial$-compressible in $\mathcal{M}$ if there is a disk $D \subset M$ such that $D \cap F=\partial D \cap F=: \gamma$ is a non-trivial arc in $\mathcal{F}$, and $\operatorname{cl}(\partial D \backslash \gamma)$ is an arc in $\partial M$. The disk $D$ is called a $\partial$-compressing disk of $\mathcal{F}$. We say that $\mathcal{F}$ is $\partial$-incompressible in $\mathcal{M}$ if $\mathcal{F}$ is not $\partial$-compressible in $\mathcal{M}$. A surface $\mathcal{F}$ is $\partial$-parallel in $\mathcal{M}$ if $F$ cuts off $\mathcal{M}^{\prime}$ from $\mathcal{M}$ with $\mathcal{M}^{\prime} \cong \mathcal{F} \times[0,1](=(F \times[0,1],(F \cap J) \times[0,1]))$.

We say that $\mathcal{M}$ is reducible if there is a 2-sphere disjoint from $J$ which does not bound a 3-ball $B^{3}$. We say that $\mathcal{M}$ is $\partial$-reducible if there is a disk $\bar{D} \subset M \backslash J$ such that $\bar{D} \cap \partial M=\partial \bar{D}$ and $\partial \bar{D}$ is essential in $\partial \mathcal{M}=(\partial M, \partial M \cap J)$. We say that $\mathcal{M}$ is $\partial$-irreducible if $\mathcal{M}$ is not $\partial$-reducible.

A 3-manifold $C$ is called a (genus $g$ ) compression body if there exists a closed surface $F$ of genus $g$ such that $C$ is obtained from $F \times[0,1]$ by attaching 2 -handles along mutually disjoint loops in $F \times\{0\}$ and filling in some resulting 2-sphere boundary components with 3 -handles. We denote $F \times\{1\}$ by $\partial_{+} C$ and $\partial C \backslash \partial_{+} C$ by $\partial_{-} C$. A compression body $C$ is called a handlebody if $\partial_{-} C=\varnothing$. The triplet $\left(C_{1}, C_{2} ; S\right)$ is called a (genus $g$ ) Heegaard splitting of $M$ if $C_{1}$ and $C_{2}$ are (genus $g$ ) compression bodies with $C_{1} \cup C_{2}=M$ and $C_{1} \cap C_{2}=\partial_{+} C_{1}=\partial_{+} C_{2}=S$.

A simple arc $\gamma$ properly embedded in a compression body $C$ is said to be vertical if $\gamma$ is isotopic to an arc with \{a point $\times[0,1] \subset \partial_{-} C \times[0,1]$. A simple arc $\gamma$ properly embedded in $C$ is said to be trivial if there is a disk $\delta$ in $C$ with $\gamma \subset \partial \delta$ and $\partial \delta \backslash \gamma \subset \partial_{+} C$. Such a disk $\delta$ is called a bridge disk of $\gamma$. A disjoint union of trivial arcs is said to be mutually trivial if they admits a disjoint union of bridge disks.

Let $L$ be a link in a connected compact orientable 3-manifold $M$. We say that $L$ admits a $(g, 0)$-bridge position if there is a genus $g$ Heegaard splitting $\left(C_{1}, C_{2} ; S\right)$ of $M$ with $L \cap S=\varnothing$ such that $\operatorname{cl}\left(C_{i} \backslash \eta\left(L ; C_{i}\right)\right)(i=1,2)$ are compression bodies. We say that $L$ admits a $(g, b)$-bridge position $(b>0)$ if there is a genus $g$ Heegaard splitting $\left(C_{1}, C_{2} ; S\right)$ of $M$ such that $C_{i} \cap L$ consists of $b$ arcs which are mutually trivial for each $i=1,2$. Set $\mathcal{C}_{i}=\left(C_{i}, C_{i} \cap L\right)$ and $\mathcal{S}=(S, S \cap L)$. We call the triplet 
$\left(\mathcal{C}_{1}, \mathcal{C}_{2} ; \mathcal{S}\right)$ a $(g, b)$-bridge splitting of $\mathcal{M}=(M, L)$ and $\mathcal{S}$ is called a $(g, b)$-bridge surface, or a bridge surface for short. We notice that a $(g, 0)$-bridge splitting of $\mathcal{M}=(M, L)$ is also called a Heegaard splitting of $\mathcal{M}$ and a $(g, 0)$-bridge surface of $\mathcal{M}$ is called a Heegaard surface of $\mathcal{M}$.

Definition 2.1 Let $K$ be a knot in a connected compact orientable 3-manifold $M$. The Heegaard genus of $K \subset M$, denoted by $\operatorname{hg}(K)$, is the minimal value $g$ such that $(M, K)$ admits a Heegaard surface of genus $g$.

\subsection{C-bodies and cH-splittings}

We recall definitions of a c-compression body and a $c$-Heegaard splitting given by Tomova [18]. In this paper, they are abbreviated as $a c-b o d y$ and $a c H$-splitting respectively.

Definition 2.2 Let $J$ be a 1 -manifold properly embedded in a connected compact orientable 3-manifold $M$. A surface $\mathcal{F}=(F, F \cap J)$ is c-compressible in $\mathcal{M}=$ $(M, J)$ if there is a disk $D \subset M$ such that $D \cap F=\partial D, \partial D$ is essential in $\mathcal{F}$ and $D$ intersects $J$ in at most one point. If $|D \cap J|=1$, then $D$ is called a cut disk of $\mathcal{F}$. We say that $\mathcal{F}$ is $c$-incompressible in $\mathcal{M}$ if $\mathcal{F}$ is not c-compressible in $\mathcal{M}$. A $c$-disk is a compressing disk or a cut disk.

Definition 2.3 Let $\mathcal{C}$ be a pair of a genus $g$ compression body $C$ and a 1 -manifold $J$ properly embedded in $C$. Then $\mathcal{C}$ is called a (genus $g$ ) $c-b o d y$ if there is a disjoint union $\mathbb{D}$ of $\mathrm{c}$-disks and bridge disks which cuts $\mathcal{C}$ into some 3 -balls and a 3-manifold homeomorphic to $\partial_{-} C \times[0,1]$ with vertical arcs. Then $\mathbb{D}$ is called a complete $c$-disk system of $\mathcal{C}$. We set $\partial_{+} \mathcal{C}=\left(\partial_{+} C, \partial_{+} C \cap J\right)$ and $\partial_{-} \mathcal{C}=\left(\partial_{-} C, \partial_{-} C \cap J\right)$.

The next two lemmas are obtained by standard innermost/outermost disk arguments.

Lemma 2.4 Let $\mathcal{C}$ be a $c$-body. Suppose that there is a compressing disk $D$ of $\mathcal{C}$ which cuts $\mathcal{C}$ into $\left(\partial_{-} C \times[0,1]\right.$, vertical arcs $)$ and $\left(B^{3}\right.$, a trivial arc $)$. Then any compressing disk of $\mathcal{C}$ is isotopic to $D$.

Lemma 2.5 Let $\mathcal{C}$ be a c-body. Suppose that there is a non-separating compressing disk (resp. a cut disk) $D$ of $\mathcal{C}$ which cuts $\mathcal{C}$ into $\left(\partial_{-} C \times[0,1]\right.$, vertical arcs). Then any non-separating compressing disk (resp. a cut disk) of $\mathcal{C}$ is isotopic to $D$.

We now recall the following obtained by Hayashi and Shimokawa [4] and by Tomova [18]. 
Lemma 2.6 (Hayashimo [4, Lemma 2.4]) Let $\mathcal{C}=(C, J)$ be a $c$-body such that each component of $J$ is trivial or vertical in $C$. If $\mathcal{F}=(F, F \cap J)$ is an incompressible, $\partial$-incompressible surface in $\mathcal{C}$, then $F$ is

(1) a 2 -sphere intersecting $J$ in 0 or 2 points,

(2) a disk intersecting $J$ in 0 or 1 points,

(3) a vertical annulus disjoint from $J$, or

(4) a closed surface parallel to a component of $\partial_{-} \mathcal{C}$.

Lemma 2.7 (Tomova [18, Corollary 3.7]) If $\mathcal{F}=(F, F \cap J)$ is a c-incompressible, d-incompressible surface in a c-body $\mathcal{C}=(C, J)$, then $F$ is

(1) a 2 -sphere intersecting $J$ in 0 or 2 points,

(2) a disk intersecting $J$ in 0 or 1 points,

(3) a vertical annulus disjoint from $J$, or

(4) a closed surface parallel to a component of $\partial_{-} \mathcal{C}$.

Definition 2.8 Let $J$ be a 1 -manifold properly embedded in a connected compact orientable 3-manifold $M$. The triplet $\left(\mathcal{C}_{1}, \mathcal{C}_{2} ; \mathcal{S}\right)$ is a (genus $g$ ) $\mathrm{cH}$-splitting of $\mathcal{M}=(M, J)$ if $\mathcal{C}_{i}\left(i=1\right.$ and 2 ) are (genus $g$ ) c-bodies with $\mathcal{C}_{1} \cup \mathcal{C}_{2}=\mathcal{M}$ and $\mathcal{C}_{1} \cap \mathcal{C}_{2}=\partial_{+} \mathcal{C}_{1}=\partial_{+} \mathcal{C}_{2}=\mathcal{S}$. The surface $\mathcal{S}$ is called a $c H$-surface of $\mathcal{M}$.

We notice that a $(g, b)$-bridge splitting of $\mathcal{M}$ is a genus $g \mathrm{cH}-$ splitting and that if $\mathcal{M}=(M, J)$ with $J=\varnothing$, then a cH-splitting is a Heegaard splitting of $M$.

Definition 2.9 Let $J$ be a 1 -manifold properly embedded in a connected compact orientable 3-manifold $M$, and let $\left(\mathcal{C}_{1}, \mathcal{C}_{2} ; \mathcal{S}\right)$ be a cH-splitting of $\mathcal{M}=(M, J)$.

(1) The cH-surface $\mathcal{S}$ is said to be $\partial$-reducible if there is a $\partial$-reducing disk $\bar{D}$ of $\mathcal{M}$ such that $\bar{D} \cap S$ is a single curve.

(2) The cH-surface $\mathcal{S}$ is said to be reducible if there are compressing disks $D_{i}$ $(i=1,2)$ of $\mathcal{C}_{i}$ with $\partial D_{1}=\partial D_{2}$. The $\mathrm{cH}$-surface $\mathcal{S}$ is said to be irreducible if it is not reducible.

(3) The cH-surface $\mathcal{S}$ is said to be stabilized if there are compressing disks $D_{i}$ $(i=1,2)$ of $\mathcal{C}_{i}$ such that $\partial D_{1}$ and $\partial D_{2}$ intersect transversely in a single point.

(4) The cH-surface $\mathcal{S}$ is said to be meridionally stabilized if there are a compressing disk $D_{i}$ of $\mathcal{C}_{i}$ and a cut disk $D_{j}$ of $\mathcal{C}_{j}((i, j)=(1,2)$ or $(2,1))$ such that $\partial D_{1}$ and $\partial D_{2}$ intersect transversely in a single point. 
(5) The $\mathrm{cH}$-surface $\mathcal{S}$ is said to be weakly reducible if there are compressing disks $D_{i}(i=1,2)$ of $\mathcal{C}_{i}$ with $\partial D_{1} \cap \partial D_{2}=\varnothing$. The $\mathrm{cH}$-surface $\mathcal{S}$ is said to be strongly irreducible if it is not weakly reducible.

(6) The cH-surface $\mathcal{S}$ is said to be $c$-weakly reducible if there are c-disks $D_{i}$ $(i=1,2)$ of $\mathcal{C}_{i}$ with $\partial D_{1} \cap \partial D_{2}=\varnothing$. The $\mathrm{cH}$-surface $\mathcal{S}$ is said to be $c$ strongly irreducible if it is not $\mathrm{c}-$ weakly reducible.

The next lemma is proved by Tomova [18].

Lemma 2.10 (Tomova [18, Theorem 6.2]) Let $J$ be a 1 -manifold properly embedded in a connected compact orientable irreducible 3-manifold $M$, and let $\left(\mathcal{C}_{1}, \mathcal{C}_{2} ; \mathcal{S}\right)$ be a cH-splitting of $\mathcal{M}=(M, J)$. If $\mathcal{M}$ is $\partial$-reducible, then $\mathcal{S}$ is $\partial$-reducible.

Corollary 2.11 Let $J$ be a 1-manifold properly embedded in a connected compact orientable irreducible 3-manifold $M$, and let $\left(\mathcal{C}_{1}, \mathcal{C}_{2} ; \mathcal{S}\right)$ be a cH-splitting of $\mathcal{M}=$ $(M, J)$. Let $\bar{D}$ be a $\partial-$ reducing disk of $\mathcal{M}$ with $|\bar{D} \cap S|=1$ and $\partial \bar{D} \subset \partial_{-} C_{i}(i=1$ or 2). Suppose that $\mathcal{C}_{i}$ admits a compressing disk. Then there is a compressing disk $D$ of $\mathcal{C}_{i}$ such that $D \cap \bar{D}=\varnothing$ and hence $\mathcal{S}$ is weakly reducible.

\subsection{C-weak reduction}

We briefly recall the operation called a c-weak reduction treated in [18]. Though we here recall c-weak reductions only for bridge splittings, such operations are applied to those for $\mathrm{cH}-$-splittings as in [18].

Let $L$ be a link in a connected compact orientable 3-manifold $M$, and let $\left(\mathcal{C}_{1}, \mathcal{C}_{2} ; \mathcal{S}\right)$ be a bridge splitting of $\mathcal{M}=(M, L)$. Suppose that $\mathcal{S}$ is c-weakly reducible. Then there are disjoint unions of c-disks of $\mathcal{C}_{i}(i=1,2)$, say $\mathbb{D}_{i}$, such that $\partial \mathbb{D}_{1} \cap \partial \mathbb{D}_{2}=\varnothing$. Since each c-disk cuts a c-body into c-bodies, we obtain a collection of c-bodies $\mathcal{C}_{11}$ by cutting $\mathcal{C}_{1}$ along $\mathbb{D}_{1}$. Let $\mathcal{C}_{12}$ be a 3 -manifold obtained from $\partial_{+} \mathcal{C}_{11} \times[0,1]$ by attaching $\eta\left(\mathbb{D}_{2} ; \mathcal{C}_{2}\right)$. We notice that $\mathcal{C}_{12}$ is also a collection of c-bodies with $\partial_{+} \mathcal{C}_{12}=\partial_{+} \mathcal{C}_{11}$. Let $\mathcal{C}_{21}$ be a 3 -manifold obtained from $\partial_{-} \mathcal{C}_{12} \times[0,1]$ by attaching $\eta\left(\mathbb{D}_{1} ; \mathcal{C}_{1}\right)$. We also notice that $\mathcal{C}_{21}$ is a collection of c-bodies with $\partial_{-} \mathcal{C}_{21}=\partial_{-} \mathcal{C}_{12}=: \mathcal{F}$. Finally, we let $\mathcal{C}_{22}$ be a collection of c-bodies by cutting $\mathcal{C}_{2}$ along $\mathbb{D}_{2}$. Set $\mathcal{M}_{i}=\mathcal{C}_{i 1} \cup \mathcal{C}_{i 2}$ for each $i=1,2$. Then we see that $\left\{\mathcal{C}_{i 1}, \mathcal{C}_{i 2}\right\}$ gives a collection of $\mathrm{cH}$-splittings of $\mathcal{M}_{i}$ for each $i=1,2$ (see Figure 2). We say that a collection of surfaces $\mathcal{F}$ is obtained by the $c$-weak reduction with respect to $\left(\mathbb{D}_{1}, \mathbb{D}_{2}\right)$. If $\mathbb{D}_{i}(i=1,2)$ are disjoint unions of compressing disks of $\mathcal{C}_{i}$ such that $\partial \mathbb{D}_{1} \cap \partial \mathbb{D}_{2}=\varnothing$, then such an operation is originally introduced by Casson and Gordon [1] and is called a weak reduction.

In this paper, we slightly modify the operation as in the following remark. 

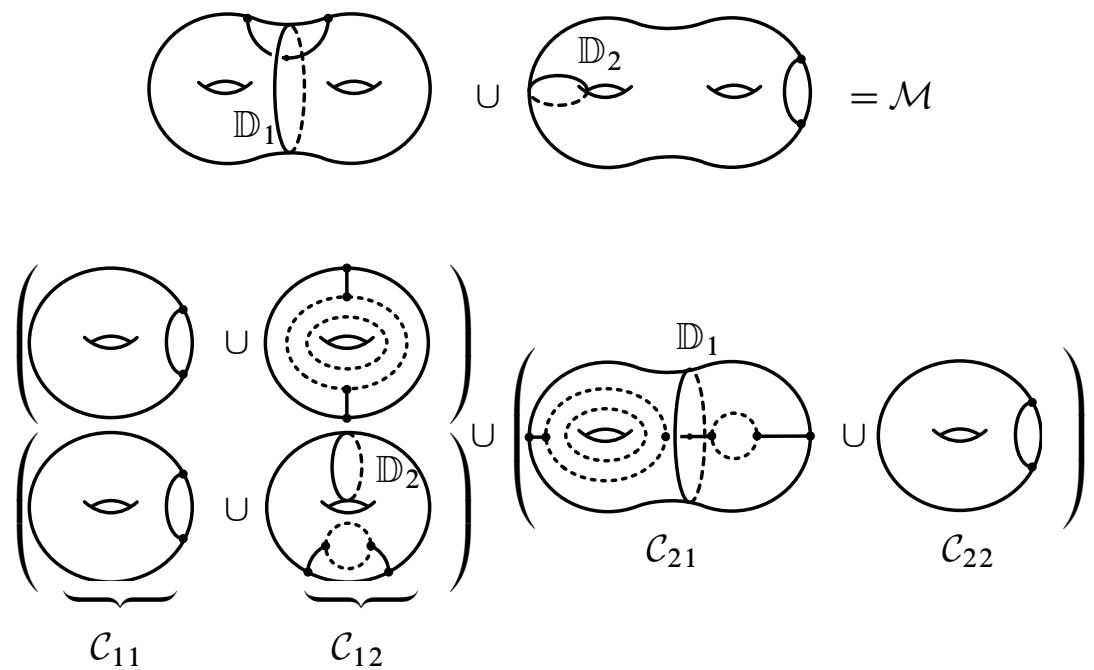

Figure 2: An example of c-weak reduction

Remark 2.12 Suppose that $\mathbb{D}_{i}$, say $i=2$, consists of a compressing disk $D_{2}$ which cuts off a 3 -ball $\mathcal{B}=(B, B \cap L)$ from $\mathcal{C}_{2}$ such that $B \cap L$ is a collection of mutually trivial arcs and that $\partial B \cap \partial \mathbb{D}_{1}=\varnothing$. Then we slightly modify the operation as follows: let $\mathcal{C}_{12}$ be a 3 -manifold obtained from $\partial_{+} \mathcal{C}_{11} \times[0,1]$ by attaching $\mathcal{B}$ along $\operatorname{cl}\left(B \backslash D_{2}\right)$, and let $\mathcal{C}_{22}$ be a 3 -manifold obtained from $\mathcal{C}_{2}$ by cutting $\mathcal{B}$ off. Then we see that $\mathcal{C}_{12}$ and $\mathcal{C}_{22}$ are $\mathrm{c}$-bodies and that $\left\{\mathcal{C}_{i 1}, \mathcal{C}_{i 2}\right\}$ similarly gives a collection of $\mathrm{cH}$-splittings of $\mathcal{M}_{i}$ for each $i=1,2$. We notice that $D_{2}$ is naturally extended to be a compressing disk $\widehat{D}_{2}$ of $\mathcal{C}_{12}$ (see, for example, Figure 3 ).

\subsection{Meridional destabilizing number}

Let $L$ be a link in a connected compact orientable 3 -manifold $M$, and let $\left(\mathcal{C}_{1}, \mathcal{C}_{2} ; \mathcal{S}\right)$ be a $(g, b)$-bridge splitting of $\mathcal{M}=(M, L)$. Suppose that $\mathcal{S}$ is meridionally stabilized. Then there are a compressing disk $D_{i}$ of $\mathcal{C}_{i}$ and a cut disk $D_{j}$ of $\mathcal{C}_{j}((i, j)=(1,2)$ or $(2,1)$, say the latter) such that $\partial D_{1}$ intersects $\partial D_{2}$ transversely in a single point. We notice that $D_{1}$ and $D_{2}$ are non-separating in $C_{1}$ and $C_{2}$ respectively. Cutting $\mathcal{C}_{1}$ along $D_{1}$, we obtain a pair $\mathcal{C}_{1}^{\prime}$ of a genus $g-1$ compression body and $b+1$ mutually trivial arcs. Gluing $\mathcal{C}_{2}$ together with $\eta\left(D_{1} ; C_{1}\right)$ containing a trivial arc, we also obtain a pair $\mathcal{C}_{2}^{\prime}$ of a genus $g-1$ compression body and $b+1$ mutually trivial arcs. Hence $\left\{\mathcal{C}_{1}^{\prime}, \mathcal{C}_{2}^{\prime}\right\}$ gives a $(g-1, b+1)$-bridge splitting of $\mathcal{M}$. Such an operation is called a meridional destabilization. 
Definition 2.13 Let $K$ be a knot in $M$. Meridional destabilizing number of $K$, denoted by $\operatorname{md}(K)$, is defined by the maximal number of $m$ such that $\mathcal{M}=(M, K)$ admits a $(\operatorname{hg}(K)-m, m)$-bridge $\operatorname{md}(K)=0$ if none of the minimal genus Heegaard splittings of $\mathcal{M}$ are meridionally stabilized.

\subsection{Connected sum}

For each $i=1,2$, let $J_{i}$ be a 1 -manifold properly embedded in a connected compact orientable 3-manifold $M_{i}$, and take a point $p_{i}$ in the interior of $J_{i}$. We notice that $\eta\left(p_{i} ; M_{i}\right) \cong B^{3}$ and $\eta\left(p_{i} ; M_{i}\right) \cap J_{i}$ is a trivial arc in $\eta\left(p_{i} ; M_{i}\right)$. Set $M_{i}^{\prime}=$ $\operatorname{cl}\left(M_{i} \backslash \eta\left(p_{i} ; M_{i}\right)\right), \partial_{0} M_{i}^{\prime}=\partial M_{i}^{\prime} \backslash \partial M_{i}$, and let $h: \partial_{0} M_{1}^{\prime} \rightarrow \partial_{0} M_{2}^{\prime}$ be a gluing map with $h\left(\partial_{0} M_{1}^{\prime} \cap J_{1}\right)=\partial_{0} M_{2}^{\prime} \cap J_{2}$ and $h_{*}\left(\left[\partial_{0} M_{1}^{\prime} \cap J_{1}\right]\right)=-\left[\partial_{0} M_{2}^{\prime} \cap J_{2}\right]$, where [ $]$ is a homology class and $h_{*}$ is the homomorphism induced by $h$. The 3-manifold $M_{1}^{\prime} \cup_{h} M_{2}^{\prime}$ is denoted by $M_{1} \# M_{2}$, and the 1 -manifold $\left(M_{1}^{\prime} \cap J_{1}\right) \cup_{h}\left(M_{2}^{\prime} \cap J_{2}\right)$ is denoted by $J_{1} \# J_{2}$. We call this operation the connected sum. If $\left(M_{i}^{\prime}, M_{i}^{\prime} \cap J_{i}\right)$ is neither (a 3-manifold, a trivial arc) nor $\left(S^{2} \times[0,1]\right.$, two vertical arcs) for each $i=1,2$, then the image $\Sigma$ of $\partial_{0} M_{i}^{\prime}$ in $M_{1} \# M_{2}$ is called a decomposing sphere of $J_{1} \# J_{2}$.

Proposition 2.14 For each $i=1,2$, let $K_{i}$ be a knot in a connected compact orientable 3-manifold $M_{i}$ which admits a $\left(g_{i}, b_{i}\right)$-bridge position. If $b_{1} \neq 0$ or $b_{2} \neq 0$, then $K_{1} \# K_{2}$ admits a $\left(g_{1}+g_{2}, b_{1}+b_{2}-1\right)$-bridge position.

Proof For each $i=1,2$, let $\left(\mathcal{V}_{i 1}, \mathcal{V}_{i 2} ; \mathcal{S}_{i}\right)$ be a $\left(g_{i}, b_{i}\right)$-bridge splitting of $\left(M_{i}, K_{i}\right)$, where $\mathcal{V}_{i j}=\left(V_{i j}, V_{i j} \cap K_{i}\right)(j=1,2)$. We first assume that each $b_{i}$ is not equal to zero. Let $\gamma_{i 2}$ be a component of $V_{i 2} \cap K_{i}$. We notice that $\gamma_{i 2}$ is trivial in $\mathcal{V}_{i 2}$ and hence $\gamma_{i 2}$ admits a bridge disk, say $\delta_{i 2}$. To take the connected sum of $K_{1}$ and $K_{2}$, we let $V_{i 2}^{\prime}$ be a 3 -manifold obtained from $V_{i 2}$ by removing $\eta\left(\gamma_{i 2} ; V_{i 2}\right)$. We notice that $V_{i 2}^{\prime} \cap \eta\left(\gamma_{i 2} ; V_{i 2}\right)$ consists of an annulus, say $A_{i 2}$ which admits a $\partial-$ compressing disk $\delta_{i 2}^{\prime}=\delta_{i 2} \cap V_{i 2}^{\prime}$. Set $\mathcal{V}_{i 2}^{\prime}=\left(V_{i 2}^{\prime}, V_{i 2} \cap K_{i}\right), M_{i}^{\prime}=V_{i 1} \cup V_{i 2}^{\prime}$ and $K_{i}^{\prime}=M_{i}^{\prime} \cap K$. Then we obtain the connected sum $K_{1} \# K_{2}$ by gluing $\left(M_{1}^{\prime}, K_{1}^{\prime}\right)$ to $\left(M_{2}^{\prime}, K_{2}^{\prime}\right)$ along a map $h$. In particular, $A_{12}$ is identified with $A_{22}$ by a map $h$. This implies that $V_{12}^{\prime} \cup_{h} V_{22}^{\prime}$ is a compression body of genus $g_{1}+g_{2}+1$ which intersects $K_{1} \# K_{2}$ in $b_{1}+b_{2}-2$ mutually trivial arcs. We also see that $V_{11} \cup_{h} V_{21}$ is a compression body of genus $g_{1}+g_{2}+1$ which intersects $K_{1} \# K_{2}$ in $b_{1}+b_{2}-2$ mutually trivial arcs. We hereafter take such a map $h$ that $A_{12} \cap \delta_{12}^{\prime}$ is identified with $A_{22} \cap \delta_{22}^{\prime}$. Set $W_{1}=V_{11} \cup_{h} V_{21}$ and $W_{2}=V_{12}^{\prime} \cup_{h} V_{22}^{\prime}$. Then $\left\{W_{1}, W_{2}\right\}$ gives a $\left(g_{1}+g_{2}+1, b_{1}+b_{2}-2\right)$-bridge splitting of $K_{1} \# K_{2}$. Since $\operatorname{cl}\left(\partial \eta\left(\gamma_{i 2} ; V_{i 2}\right) \backslash A_{i 2}\right)$ consists of two disks in $\partial V_{i 1}$, we let $D_{1} \subset W_{1}$ be a copy of such a component. Then 
$D_{1}$ is a cut disk of $W_{1}$. We notice that $D_{2}=\delta_{12}^{\prime} \cup_{h} \delta_{22}^{\prime}$ is a compressing disk of $W_{2}$ such that $\partial D_{1}$ and $\partial D_{2}$ intersect transversely in a single point. Hence the bridge splitting given by $\left\{W_{1}, W_{2}\right\}$ is meridionally stabilized and therefore $K_{1} \# K_{2}$ admits a $\left(g_{1}+g_{2}, b_{1}+b_{2}-1\right)$-bridge position.

Suppose next that $b_{1}$ or $b_{2}$, say the latter, is equal to zero. We may assume that $K_{2} \subset V_{21}$. Let $V_{12}^{\prime}$ be as above and set $V_{21}^{\prime}=\operatorname{cl}\left(V_{21} \backslash E_{21}\right)$, where $E_{21}$ is a cut disk of $V_{21}$. We reset $W_{1}=V_{11} \cup V_{21}^{\prime}$ and $W_{2}=V_{12}^{\prime} \cup_{h} V_{22}$. Then we also see that $\left\{W_{1}, W_{2}\right\}$ gives a $\left(g_{1}+g_{2}, b_{1}+b_{2}-1\right)$-bridge splitting of $K_{1} \# K_{2}$.

\section{Incompressible surfaces and cH-splittings}

Let $M$ be a connected compact orientable irreducible 3-manifold, $J$ a 1 -manifold properly embedded in $M$. Recall that we assume that $J$ is not split. We obtain the following by using a standard innermost disk argument.

Lemma 3.1 Let $J$ be a 1-manifold properly embedded in a connected compact orientable irreducible 3-manifold $M$. Let $\mathcal{F}=(F, F \cap J)$ and $\mathcal{F}^{\prime}=\left(F^{\prime}, F^{\prime} \cap J\right)$ be closed surfaces incompressible in $\mathcal{M}=(M, J)$. Then there is a closed incompressible surface $\mathcal{F}^{\prime \prime}=\left(F^{\prime \prime}, F^{\prime \prime} \cap J\right)$ with $F^{\prime \prime} \cong F^{\prime}$ and $\left|F^{\prime \prime} \cap J\right|=\left|F^{\prime} \cap J\right|$ such that $F^{\prime \prime} \cap F=\varnothing$ or that each component of $F^{\prime \prime} \cap F$ is non-trivial both in $\mathcal{F}^{\prime \prime}$ and in $\mathcal{F}$. Moreover, $\mathcal{F}^{\prime \prime}$ is ambient isotopic to $\mathcal{F}^{\prime}$ in $\mathcal{M}$ if $M=S^{3}$.

Remark 3.2 To prove Lemma 3.1 by using a standard innermost disk argument, we suppose that $F \cap F^{\prime} \neq \varnothing$ and there is a component $\alpha$ of $F \cap F^{\prime}$ which is trivial in $\mathcal{F}$ or $\mathcal{F}^{\prime}$. Then we notice that $\alpha$ must be trivial both in $\mathcal{F}$ and in $\mathcal{F}^{\prime}$ because $M$ is irreducible and $J$ is properly embedded in $M$.

The following is essentially obtained by Schultens [17] (see also Morimoto [12]).

Lemma 3.3 (Schultens [17, Lemma 6]) Let $J$ be a 1-manifold properly embedded in a connected compact orientable irreducible 3-manifold $M$, and let $\left(\mathcal{C}_{1}, \mathcal{C}_{2} ; \mathcal{S}\right)$ be a $\mathrm{cH}$-splitting of $\mathcal{M}=(M, J)$. Suppose that $\mathcal{S}$ is strongly irreducible and that $J$ admits a decomposing sphere. Then there is a decomposing sphere $\Sigma$ of $J$ such that each component of $\Sigma \cap S$ is non-trivial in $(\Sigma, J \cap \Sigma)$ and is essential in $\mathcal{S}$.

Proof Morimoto's argument in the proof of [12, Lemma 2.3] will work here. Recall that $\mathcal{C}_{i}=\left(C_{i}, C_{i} \cap J\right)(i=1,2)$ and $\mathcal{S}=(S, S \cap J)$. We assume that $C_{1}$ is small enough to intersect a decomposing sphere $\Sigma$ only in c-disks of $\mathcal{C}_{1}$. Set $\Sigma_{i}=\Sigma \cap \mathcal{C}_{i}$ 
( $i=1,2)$. We take a decomposing sphere $\Sigma$ so that $\left(\left|\Sigma_{1}\right|,\left|\Sigma_{2} \cap J\right|\right)$ is lexicographically minimal among all such decomposing spheres. Then each component of $\Sigma_{2}^{(0)}:=\Sigma_{2}$ is c-incompressible in $\mathcal{C}_{2}$. Hence we have a sequence of $\partial$-compressions for $\Sigma_{2}$ which realizes a hierarchy $\left\{\left(\Sigma_{2}^{(j)}, a_{j}\right)\right\}_{0 \leq j \leq n}$, that is, $a_{j}$ is a non-trivial arc in $\Sigma_{2}^{(j)}$, $\Sigma_{2}^{(j+1)}$ is obtained by cutting $\Sigma_{2}^{(j)}$ along $a_{j}$, and $\Sigma_{2}^{(n)}$ consists of c-disks of $\mathcal{C}_{2}$. Set $\Sigma_{1}^{(j)}=\operatorname{cl}\left(\Sigma \backslash \Sigma_{2}^{(j)}\right)$. By the minimality, we may also assume that each component of $\Sigma_{1}^{(j)} \cap S$ is essential in $\mathcal{S}$ for any integer $j$ with $0 \leq j \leq n$.

If $\Sigma_{1}^{(0)}$ or $\Sigma_{2}^{(n)}$ consists of cut disks of $\mathcal{C}_{1}$ or $\mathcal{C}_{2}$ respectively, then we are done. Hence we assume that both $\Sigma_{1}^{(0)}$ and $\Sigma_{2}^{(n)}$ contain compressing disks. Let $k$ be the maximal integer such that $\Sigma_{1}^{(k)}$ contains a compressing disk, say $D_{1}$, of $\mathcal{C}_{1}$. Suppose that $\Sigma_{2}^{(k+1)}$ contains a compressing disk, say $D_{2}$, of $\mathcal{C}_{2}$. We notice that $D_{2}$ is obtained by cutting an annulus component, say $A_{2}$, of $\Sigma_{2}^{(k)}$ along $a_{k}$. It follows from strong irreducibility of $\mathcal{S}$ that $\partial D_{1}$ is a component of $\partial A_{2}$. Taking a parallel copy, say $D_{2}^{\prime}$, of $D_{2}$ in $\mathcal{C}_{2}$ with $A_{2} \cap D_{2}^{\prime}=\varnothing$, we see that $\partial D_{1} \cap \partial D_{2}^{\prime}=\varnothing$, contradicting strong irreducibility of $\mathcal{S}$. Therefore $\Sigma_{2}^{(k+1)}$ contains no compressing disks of $\mathcal{C}_{2}$ and hence we have the desired result because $\Sigma_{1}^{(k+1)}$ also contains no compressing disks of $\mathcal{C}_{1}$.

Corollary 3.4 Let $J$ be a 1-manifold properly embedded in a connected compact orientable irreducible 3 -manifold $M$, and let $\left(\mathcal{C}_{1}, \mathcal{C}_{2} ; \mathcal{S}\right)$ be a $\mathrm{cH}$-splitting of $\mathcal{M}=$ $(M, J)$. Suppose that $\mathcal{S}$ is strongly irreducible and that $J$ admits a decomposing sphere. Then either

(1) there are separating cut disks $E_{1}$ and $E_{2}$ of $\mathcal{C}_{1}$ and $\mathcal{C}_{2}$ respectively with $\partial E_{i}=$ $\partial E_{j}$, or

(2) there is a cut disk $E_{i}$ of $\mathcal{C}_{i}$ and a compressing disk $E_{j}$ of $\mathcal{C}_{j}$ such that $\partial E_{i} \cap$ $\partial E_{j}=\varnothing$ for $(i, j)=(1,2)$ or $(2,1)$.

Proof It follows from Lemma 3.3 that there is a decomposing sphere $\Sigma$ of $J$ such that each component of $\Sigma \cap S$ is essential in $\mathcal{S}$, and the components of $\Sigma$ cut along $\Sigma \cap S$ consist of two disks $\Delta$ and $\Delta^{\prime}$ with $|\Delta \cap J|=\left|\Delta^{\prime} \cap J\right|=1$ and possibly annuli disjoint from $J$. Without loss of generality, we assume that $\Delta$ is a cut disk of $\mathcal{C}_{1}$. If $\Sigma \cap C_{2}$ contains no annulus components, then $\Sigma \cap C_{1}$ consists of the disk $\Delta$ and $\Sigma \cap C_{2}$ similarly consists of the disk $\Delta^{\prime}$. Hence we have the conclusion (1) of Corollary 3.4. Otherwise, $\Sigma \cap C_{2}$ contains an annulus component which is $\partial$-compressible in $\mathcal{C}_{2}$. Let $A$ be an annulus component of $\Sigma \cap C_{2}$ such that a $\partial$-compressing disk $\delta$ of $A$ is disjoint from the other components of $\Sigma \cap C_{2}$. After the $\partial$-compression along $\delta$, we have a compressing disk $D_{2}$ of $\mathcal{C}_{2}$. A parallel copy of $\Delta$ and $D_{2}$ satisfy the conclusion (2) of Corollary 3.4. 
Corollary 3.5 Let $L$ be a link in a connected compact orientable irreducible 3manifold $M$, and let $\left(\mathcal{C}_{1}, \mathcal{C}_{2} ; \mathcal{S}\right)$ be a bridge splitting of $\mathcal{M}=(M, L)$. Suppose that $\mathcal{S}$ is strongly irreducible and that $L$ admits a decomposing sphere. Then there are a non-separating cut disk $E_{i}$ of $\mathcal{C}_{i}$ and a compressing disk $E_{j}$ of $\mathcal{C}_{j}$ such that $\partial E_{i} \cap \partial E_{j}=\varnothing$ for $(i, j)=(1,2)$ or $(2,1)$.

Proof By assumption, we have one of the conclusions of Corollary 3.4. If the conclusion (2) of Corollary 3.4 holds, then we may assume that there are a cut disk $E_{1}$ of $\mathcal{C}_{1}$ and a compressing disk $E_{2}$ of $\mathcal{C}_{2}$ such that $\partial E_{1} \cap \partial E_{2}=\varnothing$. Suppose, towards a contradiction, $E_{1}$ is separating in $C_{1}$. Then $E_{1}$ cuts $C_{1}$ into two compression bodies $C_{11}$ and $C_{12}$. Let $\beta_{1}$ be the component of $C_{1} \cap L$ with $\beta_{1} \cap E_{1} \neq \varnothing$. Then $C_{11}$ (resp. $C_{12}$ ) contains $\beta_{1} \cap C_{11}$ (resp. $\beta_{1} \cap C_{12}$ ) as a trivial arc. Since $\partial E_{1}$ is essential in $\mathcal{S}$, we see that there are compressing disks $D_{11}$ and $D_{12}$ of $\mathcal{C}_{11}=\left(C_{11}, C_{11} \cap L\right)$ and $\mathcal{C}_{12}=\left(C_{12}, C_{12} \cap L\right)$ respectively. We notice that $D_{11}$ and $D_{12}$ are also compressing disks of $\mathcal{C}_{1}$.

We now suppose that the conclusion (1) of Corollary 3.4 holds. Then we similarly see that $E_{2}$ cuts $C_{2}$ into two compression bodies $C_{21}$ and $C_{22}$ and that there are compressing disks $D_{21}$ and $D_{22}$ of $\mathcal{C}_{21}=\left(C_{21}, C_{21} \cap L\right)$ and $\mathcal{C}_{22}=\left(C_{22}, C_{22} \cap L\right)$. Since $\partial E_{1}=\partial E_{2}$, we have either $\partial D_{11} \cap \partial D_{21}=\varnothing$ or $\partial D_{12} \cap \partial D_{21}=\varnothing$, contradicting strong irreducibility of $\mathcal{S}$. If the conclusion (2) of Corollary 3.4 holds, then we also see that $\partial D_{11} \cap \partial E_{2}=\varnothing$ or $\partial D_{12} \cap \partial E_{2}=\varnothing$. This again contradicts strong irreducibility of $\mathcal{S}$. Therefore $E_{1}$ is non-separating in $C_{1}$ and we have the desired conclusion.

\section{$4(1,2)$-bridge splittings}

Theorem 4.1 Let $K$ be a knot in $S^{3}$ and $\left(\mathcal{C}_{1}, \mathcal{C}_{2} ; \mathcal{S}\right)$ a $(1,2)$-bridge splitting of $\left(S^{3}, K\right)$. Suppose that $\mathcal{S}$ is strongly irreducible and that $K$ admits a decomposing sphere. Then one of the following holds.

(1) $\mathcal{S}$ is meridionally stabilized.

(2) There is a c-weak reduction yielding a 2-sphere which intersects $K$ in four points and is incompressible in $\left(S^{3}, K\right)$.

Proof Since $\mathcal{S}$ is strongly irreducible and $K$ admits a decomposing sphere, we have the conclusion of Corollary 3.5. Without loss of generality, we assume that there are a non-separating cut disk $E_{1}$ of $\mathcal{C}_{1}$ and a compressing disk $E_{2}$ of $\mathcal{C}_{2}$ such that $\partial E_{1} \cap \partial E_{2}=\varnothing$. Then we have:

(i) $E_{2}$ is a non-separating compressing disk of $\mathcal{C}_{2}$, 
(ii) $E_{2}$ cuts off a 3-ball with two mutually trivial $\operatorname{arcs}$ from $\mathcal{C}_{2}$, or

(iii) $E_{2}$ cuts off a $3-$ ball with a single trivial arc from $\mathcal{C}_{2}$.

If we have the condition (i), then $C_{1} \cup C_{2}$ contains a non-separating 2-sphere, a contradiction. The condition (ii) implies that $\mathcal{S}$ is meridionally stabilized. Hence we consider the condition (iii). We now do the c-weak reduction with respect to $\left(E_{1}, E_{2}\right)$ (see Figure 3). We notice that $\mathcal{C}_{11}$ is a c-body obtained by cutting $\mathcal{C}_{1}$ along $E_{1}, \mathcal{C}_{12}$ is a c-body obtained from $\partial_{+} \mathcal{C}_{11} \times[0,1]$ by attaching a 3 -ball $\mathcal{B}$ with a trivial arc which is obtained by cutting $\mathcal{C}_{2}$ along $E_{2}, \mathcal{C}_{21}$ is a c-body obtained from $\partial_{-} \mathcal{C}_{12} \times[0,1]$ by attaching $\eta\left(E_{1} ; C_{1}\right)$ with a trivial arc, and $\mathcal{C}_{22}$ is a c-body obtained from $\mathcal{C}_{2}$ by cutting $\mathcal{B}$ off. We notice that $E_{2}$ is naturally extended to a compressing disk $\widehat{E}_{2}$ of $\mathcal{C}_{12}$. Set $\mathcal{M}_{i}=\mathcal{C}_{i 1} \cup \mathcal{C}_{i 2}, \mathcal{S}_{i}=\mathcal{C}_{i 1} \cap \mathcal{C}_{i 2}$ for each $i=1,2$ and $\mathcal{F}=\partial_{-} \mathcal{C}_{12}=\partial_{-} \mathcal{C}_{21}$. If both $\mathcal{M}_{1}$ and $\mathcal{M}_{2}$ are $\partial$-irreducible, then $\mathcal{F}$ is incompressible in $\left(S^{3}, K\right)$ and hence we have the conclusion (2) of Theorem 4.1. Hence we assume that $\mathcal{M}_{1}$ or $\mathcal{M}_{2}$ is $\partial$-reducible.

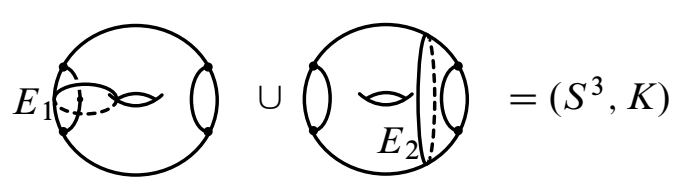

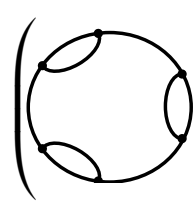

$\mathcal{C}_{11}$

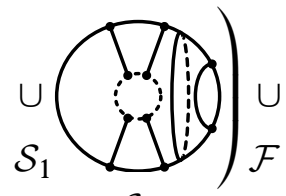

$\mathcal{C}_{12}$

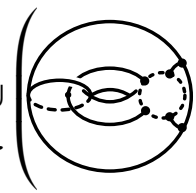

$\mathcal{C}_{21}$

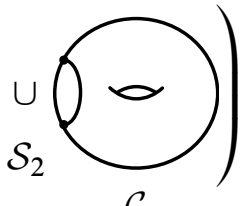

$\mathcal{C}_{22}$

Figure 3: The c-weak reduction with respect to $\left(E_{1}, E_{2}\right)$

If $\mathcal{M}_{1}$ is $\partial$-reducible, then there is a $\partial$-reducing disk $\bar{D}_{1}$ with $\left|\bar{D}_{1} \cap S_{1}\right|=1$ by Lemma 2.10, and there is a compressing disk $D_{12}$ of $\mathcal{C}_{12}$ with $D_{12} \cap \bar{D}_{1}=\varnothing$ by Corollary 2.11. It follows from Lemma 2.4 that $D_{12}$ is isotopic to $\widehat{E}_{2}$ in $\mathcal{C}_{12}$. Hence we see that $\bar{D}_{1} \cap \widehat{E}_{2}=\varnothing$. Since $\bar{D}_{1}$ can be regarded as a compressing disk of $\mathcal{C}_{1}$ and $E_{2}$ is contained in $\widehat{E}_{2}$, we see that $\mathcal{S}$ is weakly reducible, a contradiction.

If $\mathcal{M}_{2}$ is $\partial$-reducible, then it follows from Lemma 2.10 that there is a $\partial$-reducing disk $\bar{D}_{2}$ with $\left|\bar{D}_{2} \cap S_{2}\right|=1$, that is, $\bar{D}_{2}$ intersects $C_{21}$ in a vertical annulus in $C_{21}$. Since $\mathcal{C}_{21}$ is ambient isotopic to a regular neighborhood of $\partial_{-} C_{21} \cup\left(C_{21} \cap K\right)$, we see that $\bar{D}_{2}$ is isotoped to be disjoint from $E_{1}$. The disk $\bar{D}_{2}$ can be regarded as a compressing disk of $\mathcal{C}_{2}$ which is disjoint from $E_{2}$ and is not parallel to $E_{2}$. We 
notice that $\partial E_{1} \cap \partial\left(E_{2} \cup \bar{D}_{2}\right)=\varnothing$. Since $\bar{D}_{2}$ is separating in $C_{2}$, we see that $\mathcal{S}$ is meridionally stabilized.

Theorem 4.2 Let $K$ be a knot in $S^{3}$ and $\left(\mathcal{C}_{1}, \mathcal{C}_{2} ; \mathcal{S}\right)$ a $(1,2)$-bridge splitting of $\left(S^{3}, K\right)$. Suppose that $\mathcal{S}$ is weakly reducible. Then $K=K_{1} \# K_{2}$ such that $K_{1}$ admits a $(0,2)$-bridge position and $K_{2}$ admits a $(1,1)$-bridge position.

Proof Let $D_{1}$ and $D_{2}$ be compressing disks of $\mathcal{C}_{1}$ and $\mathcal{C}_{2}$ respectively such that $\partial D_{1} \cap \partial D_{2}=\varnothing$. For each $i=1,2$, we have:

(i) $D_{i}$ is a non-separating compressing disk of $\mathcal{C}_{i}$,

(ii) $D_{i}$ cuts off a 3-ball with two mutually trivial $\operatorname{arcs}$ from $\mathcal{C}_{i}$, or

(iii) $D_{i}$ cuts off a 3 -ball with a single trivial arc from $\mathcal{C}_{i}$.

Suppose first that $D_{1}$ satisfies the condition (i). If $D_{2}$ also satisfies the condition (i), then $C_{1} \cup C_{2}$ contains a non-separating 2-sphere, a contradiction. If $D_{2}$ satisfies the condition (ii), then we see that $K$ admits a $(0,2)$-bridge position and hence we have the desired conclusion. Suppose that $D_{2}$ satisfies the condition (iii). Then we have the desired conclusion by extraction operation as follows. We first notice that $D_{2}$ cuts $\mathcal{C}_{2}$ into a solid torus $\mathcal{C}_{2}^{\prime}$ with a trivial arc and a 3 -ball $\mathcal{C}_{2}^{\prime \prime}$ with a trivial arc. Attaching $\eta\left(D_{1} ; C_{1}\right)$ to $\mathcal{C}_{2}^{\prime}$, we have a 3 -ball $B$ with a properly embedded arc $J$. We notice that $(B, J)$ forms $S^{3}$ with a knot, say $K^{\prime}$, which admits a $(1,1)$-bridge position after gluing a 3 -ball with a trivial arc along their boundaries. Let $K^{\prime \prime}$ be a knot obtained from $K$ by replacing $J$ with a trivial arc in $B$. Then we see that $K^{\prime \prime}$ admits a $(0,2)$-bridge position. Hence we see that $K=K^{\prime} \# K^{\prime \prime}$ such that $K^{\prime}$ admits a $(1,1)$-bridge position and $K^{\prime \prime}$ admits a $(0,2)$-bridge position.

Suppose next that $D_{1}$ satisfies the condition (ii). If $D_{2}$ also satisfies the condition (ii), then we see that $K$ admits a $(0,2)$-bridge position and hence we have the desired conclusion. If $D_{2}$ satisfies the condition (iii), then there is a non-separating compressing disk of $\mathcal{C}_{1}$ which is disjoint from $D_{2}$ and hence we are done.

The other case is that both $D_{1}$ and $D_{2}$ satisfy the condition (iii). However, this implies that $K$ consists of two components, a contradiction.

\section{5 (1, 3)-bridge splittings}

Theorem 5.1 Let $K$ be a knot in $S^{3}$ and $\left(\mathcal{C}_{1}, \mathcal{C}_{2} ; \mathcal{S}\right)$ a $(1,3)$-bridge splitting of $\left(S^{3}, K\right)$. Suppose that $\mathcal{S}$ is strongly irreducible and that $K$ admits a decomposing sphere. Then one of the following holds. 
(1) $\mathcal{S}$ is meridionally stabilized.

(2) There is a c-weak reduction yielding a 2 -sphere which intersects $K$ in four or six points and is incompressible in $\left(S^{3}, K\right)$.

Proof Since $\mathcal{S}$ is strongly irreducible and $K$ admits a decomposing sphere, we have the conclusion of Corollary 3.5. Without loss of generality, we assume that there are a non-separating cut disk $E_{1}$ of $\mathcal{C}_{1}$ and a compressing disk $E_{2}$ of $\mathcal{C}_{2}$ such that $\partial E_{1} \cap \partial E_{2}=\varnothing$. Then we have:

(i) $E_{2}$ is a non-separating compressing disk of $\mathcal{C}_{2}$,

(ii) $E_{2}$ cuts off a 3-ball with three mutually trivial $\operatorname{arcs}$ from $\mathcal{C}_{2}$,

(iii) $E_{2}$ cuts off a 3-ball with two mutually trivial $\operatorname{arcs}$ from $\mathcal{C}_{2}$, or

(iv) $E_{2}$ cuts off a 3-ball with a single trivial arc from $\mathcal{C}_{2}$.

If we have the condition (i), then $C_{1} \cup C_{2}$ contains a non-separating 2-sphere, a contradiction. The condition (ii) implies that $\mathcal{S}$ is meridionally stabilized. The conditions (iii) and (iv) are very similar to the condition (iii) in the proof of Theorem 4.1. As in the proof of Theorem 4.1, we can do the c-weak reduction with respect to $\left(E_{1}, E_{2}\right)$ to obtain $\mathcal{M}_{i}=\mathcal{C}_{i 1} \cup \mathcal{C}_{i 2}, \mathcal{S}_{i}=\mathcal{C}_{i 1} \cap \mathcal{C}_{i 2}$ for each $i=1,2$ and $\mathcal{F}=\partial_{-} \mathcal{C}_{12}=\partial_{-} \mathcal{C}_{21}$. Then $\mathcal{F}$ is a 2 -sphere which intersects $K$ in four or six points depending on the conditions. If both $\mathcal{M}_{1}$ and $\mathcal{M}_{2}$ are $\partial$-irreducible, then $\mathcal{F}$ is incompressible in $\left(S^{3}, K\right)$ and hence we have the conclusion (2) of Theorem 5.1. Hence we assume that $\mathcal{M}_{1}$ or $\mathcal{M}_{2}$ is $\partial$-reducible.

If $\mathcal{M}_{1}$ is $\partial$-reducible, then it follows from Corollary 2.11 that $\mathcal{S}_{1}$ is weakly reducible. This implies that $\mathcal{S}$ is weakly reducible, a contradiction.

If $\mathcal{M}_{2}$ is $\partial$-reducible, then it follows from Lemma 2.10 that there is a $\partial$-reducing disk $\bar{D}_{2}$ with $\left|\bar{D}_{2} \cap S_{2}\right|=1$, that is, $\bar{D}_{2}$ intersects $C_{21}$ in a vertical annulus in $C_{21}$. Since $\mathcal{C}_{21}$ is ambient isotopic to a regular neighborhood of $\partial_{-} C_{21} \cup\left(C_{21} \cap K\right)$, we see that $\bar{D}_{2}$ is isotoped to be disjoint from $E_{1}$. The disk $\bar{D}_{2}$ can be regarded as a compressing disk of $\mathcal{C}_{2}$ which is disjoint from $E_{2}$ and is not parallel to $E_{2}$. We notice that $\partial E_{1} \cap \partial\left(\bar{D}_{2} \cup E_{2}\right)=\varnothing$. If $\bar{D}_{2}$ is non-separating in $C_{2}$, then $C_{1} \cup C_{2}$ contains a non-separating 2-sphere, a contradiction. Hence $\bar{D}_{2}$ is separating in $C_{2}$. If we have the condition (iii), then we obtain the conclusion (1) of Theorem 5.1. Suppose that we have the condition (iv). If $\bar{D}_{2}$ cuts off a 3 -ball with a single trivial arc, then we can find a compressing disk of $\mathcal{C}_{2}$ which satisfies the condition (iii) and is disjoint from $E_{1}$ by taking, if necessary, band-sum of $E_{2}$ and $\bar{D}_{2}$ disjoint from $\partial E_{1}$. If $\bar{D}_{2}$ cuts off a 3-ball with two mutually trivial arcs, then we obtain the conclusion (1) of Theorem 5.1.

This completes the proof of Theorem 5.1. 
Theorem 5.2 Let $K$ be a knot in $S^{3}$ and $\left(\mathcal{C}_{1}, \mathcal{C}_{2} ; \mathcal{S}\right)$ a $(1,3)$-bridge splitting of $\left(S^{3}, K\right)$. Suppose that $\mathcal{S}$ is weakly reducible. Then one of the following holds.

(1) $K=K_{1} \# K_{2}$ such that $K_{1}$ admits a $(0,2)$-bridge position and $K_{2}$ admits a $(1,2)$-bridge position.

(2) $K=K_{1} \# K_{2}$ such that $K_{1}$ admits a $(0,3)$-bridge position and $K_{2}$ admits a $(1,1)$-bridge position.

(3) There is a weak reduction yielding a 2-sphere which intersects $K$ in four points and is incompressible in $\left(S^{3}, K\right)$.

(4) There is a weak reduction yielding a torus which intersects $K$ in two points and is incompressible in $\left(S^{3}, K\right)$.

Proof Let $D_{1}$ and $D_{2}$ be compressing disks of $\mathcal{C}_{1}$ and $\mathcal{C}_{2}$ respectively such that $\partial D_{1} \cap \partial D_{2}=\varnothing$. For each $i=1,2$, we have:

(i) $D_{i}$ is a non-separating compressing disk of $\mathcal{C}_{i}$,

(ii) $D_{i}$ cuts off a 3-ball with three mutually trivial $\operatorname{arcs}$ from $\mathcal{C}_{i}$,

(iii) $D_{i}$ cuts off a 3-ball with two mutually trivial $\operatorname{arcs}$ from $\mathcal{C}_{i}$, or

(iv) $D_{i}$ cuts off a 3-ball with a single trivial arc from $\mathcal{C}_{i}$.

Case 1 The disk $D_{1}$ satisfies the condition (i).

If $D_{2}$ also satisfies the condition (i), then $C_{1} \cup C_{2}$ contains a non-separating 2-sphere, a contradiction. If $D_{2}$ satisfies the condition (ii), then we see that $K$ admits a $(0,3)-$ bridge position and hence we have the conclusion (2) of Theorem 5.2. If $D_{2}$ satisfies the condition (iii), then we also have the conclusion (2) of Theorem 5.2 by extraction operation (see the proof of Theorem 4.2). We therefore suppose that $D_{2}$ satisfies the condition (iv). We obtain $\mathcal{M}_{i}=\mathcal{C}_{i 1} \cup \mathcal{C}_{i 2}, \mathcal{S}_{i}=\mathcal{C}_{i 1} \cap \mathcal{C}_{i 2}$ for each $i=1,2$ and $\mathcal{F}=\partial_{-} \mathcal{C}_{12}=\partial_{-} \mathcal{C}_{21}$ by the weak reduction with respect to $\left(D_{1}, D_{2}\right)$. We notice that $\mathcal{F}$ is a 2 -sphere intersecting $K$ in four points. If both $\mathcal{M}_{1}$ and $\mathcal{M}_{2}$ are $\partial$-irreducible, then we have the conclusion (3) of Theorem 5.2. Hence we assume that $\mathcal{M}_{1}$ or $\mathcal{M}_{2}$ is $\partial$-reducible.

If $\mathcal{M}_{1}$ is $\partial$-reducible, then there is a $\partial$-reducing disk $\bar{D}_{1}$ with $\left|\bar{D}_{1} \cap S_{1}\right|=1$ by Lemma 2.10, and there is a compressing disk $D_{12}$ of $\mathcal{C}_{12}$ with $D_{12} \cap \bar{D}_{1}=\varnothing$ by Corollary 2.11. It follows from Lemma 2.4 that $D_{12}$ is isotopic to $\widehat{D}_{2}$ in $\mathcal{C}_{12}$, where $\widehat{D}_{2}$ is a compressing disk of $\mathcal{C}_{12}$ which is obtained by extending $D_{2}$ naturally. Hence we see that $\bar{D}_{1} \cap \widehat{D}_{2}=\varnothing$. The disk $\bar{D}_{1}$ can be regarded as a compressing disk of $\mathcal{C}_{1}$ which is disjoint from $D_{1}$ and is not parallel to $D_{1}$. We notice that $\partial\left(D_{1} \cup \bar{D}_{1}\right) \cap \partial D_{2}=\varnothing$. 
Whether $\bar{D}_{1}$ is separating or non-separating in $C_{1}$, we have the conclusion (1) of Theorem 5.2 by extraction operation.

If $\mathcal{M}_{2}$ is $\partial$-reducible, then there is a $\partial$-reducing disk $\bar{D}_{2}$ with $\left|\bar{D}_{2} \cap S_{2}\right|=1$ by Lemma 2.10, and there is a compressing disk $D_{21}$ of $\mathcal{C}_{21}$ with $D_{21} \cap \bar{D}_{2}=\varnothing$ by Corollary 2.11. We may assume that $D_{21}$ is non-separating in $C_{21}$. It follows from Lemma 2.5 that $D_{21}$ is isotopic to $D_{1}$ in $\mathcal{C}_{21}$. Hence we see that $D_{1} \cap \bar{D}_{2}=\varnothing$. The disk $\bar{D}_{2}$ can be regarded as a compressing disk of $\mathcal{C}_{2}$ which is disjoint from $D_{2}$ and is not parallel to $D_{2}$. We notice that $\partial D_{1} \cap \partial\left(D_{2} \cup \bar{D}_{2}\right)=\varnothing$. Since $\bar{D}_{2}$ is separating in $C_{2}, \bar{D}_{2}$ separates two arcs $C_{22} \cap K$ (Figure 4 (a) or (b)) or not (Figure 4 (c) or (d)). In each case, we have the conclusion (2) of Theorem 5.2.

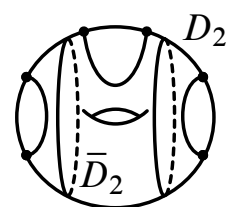

(a)

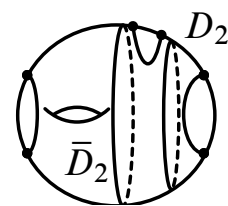

(b)

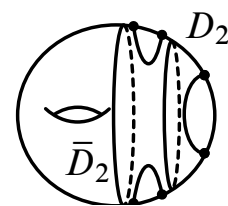

(c)

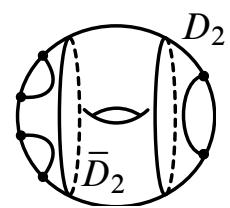

(d)

Figure 4: Possible positions of $D_{2} \cup \bar{D}_{2}$ in $\mathcal{C}_{22}$

Case 2 The disk $D_{1}$ satisfies the condition (ii).

If $D_{2}$ also satisfies the condition (ii), then we see that $K$ admits a $(0,3)$-bridge position. If $D_{2}$ satisfies the condition (iii) or (iv), then there is a non-separating compressing disk of $\mathcal{C}_{1}$ disjoint from $D_{2}$ and hence we are done in Case 1.

Case 3 The disk $D_{1}$ satisfies the condition (iii).

If $D_{2}$ also satisfies the condition (iii), then we see that $K$ is not connected, a contradiction. Hence $D_{2}$ satisfies the condition (iv) and therefore we have the conclusion (2) of Theorem 5.2 by extraction operation (see the proof of Theorem 4.2).

Case 4 The disk $D_{1}$ satisfies the condition (iv).

Then it suffices to consider the case that $D_{2}$ also satisfies the condition (iv). We obtain $\mathcal{M}_{i}=\mathcal{C}_{i 1} \cup \mathcal{C}_{i 2}, \mathcal{S}_{i}=\mathcal{C}_{i 1} \cap \mathcal{C}_{i 2}$ for each $i=1,2$ and $\mathcal{F}=\partial_{-} \mathcal{C}_{12}=\partial_{-} \mathcal{C}_{21}$ by the weak reduction with respect to $\left(D_{1}, D_{2}\right)$ (see Figure 5). We notice that $\mathcal{F}$ is a torus intersecting $K$ in two points. If both $\mathcal{M}_{1}$ and $\mathcal{M}_{2}$ are $\partial$-irreducible, then we have the conclusion (4) of Theorem 5.2. Hence we may assume that $\mathcal{M}_{2}$ is $\partial$-reducible 

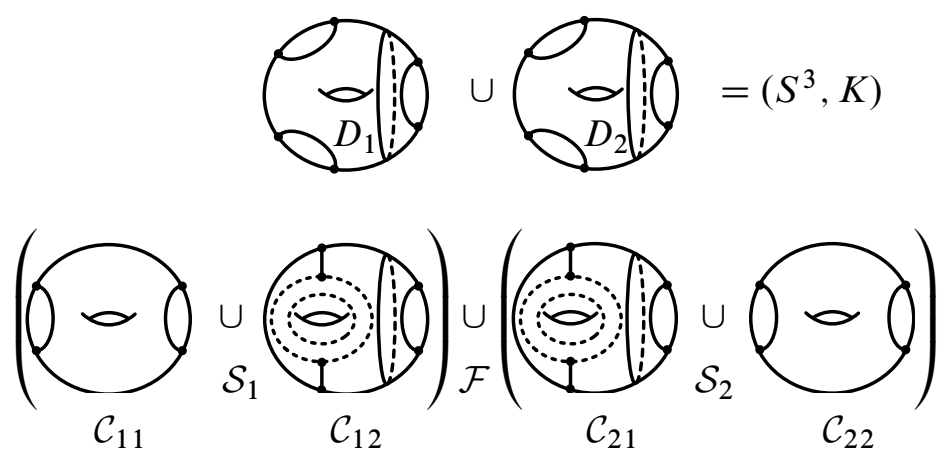

Figure 5: The weak reduction with respect to $\left(D_{1}, D_{2}\right)$

without loss of generality. Then there is a $\partial$-reducing disk $\bar{D}_{2}$ with $\left|\bar{D}_{2} \cap S_{2}\right|=1$ by Lemma 2.10 , and there is a compressing disk $D_{21}$ of $\mathcal{C}_{21}$ with $D_{21} \cap \bar{D}_{2}=\varnothing$ by Corollary 2.11. It follows from Lemma 2.4 that $D_{21}$ is isotopic to $\widehat{D}_{1}$ in $\mathcal{C}_{21}$, where $\widehat{D}_{1}$ is a compressing disk of $\mathcal{C}_{21}$ which is obtained by extending $D_{1}$ naturally. Hence we see that $\widehat{D}_{1} \cap \bar{D}_{2}=\varnothing$. The disk $\bar{D}_{2}$ can be regarded as a compressing disk of $\mathcal{C}_{2}$ which is disjoint from $D_{2}$ and is not parallel to $D_{2}$. We notice that $\partial D_{1} \cap \partial\left(\bar{D}_{2} \cup D_{2}\right)=\varnothing$. If $\bar{D}_{2}$ is non-separating in $C_{2}$, then we have the conclusion (1) of Theorem 5.2. Hence we assume that $\bar{D}_{2}$ is separating in $C_{2}$. If $\bar{D}_{2}$ separates two $\operatorname{arcs} C_{22} \cap K$, then this implies that $K$ is not connected, a contradiction. Hence $D_{2} \cup \bar{D}_{2}$ is as illustrated in Figure 4 (c) or (d), and therefore we also have the conclusion (1) of Theorem 5.2.

This completes the proof of Theorem 5.2.

\section{$6(2,2)$-bridge splittings}

Theorem 6.1 Let $K$ be a knot in $S^{3}$ and $\left(\mathcal{C}_{1}, \mathcal{C}_{2} ; \mathcal{S}\right)$ a $(2,2)$-bridge splitting of $\left(S^{3}, K\right)$. Suppose that $\mathcal{S}$ is strongly irreducible and that $K$ admits a decomposing sphere. Then one of the following holds.

(1) $\mathcal{S}$ is meridionally stabilized.

(2) There is a c-weak reduction yielding a $2-$ sphere which intersects $K$ in four or six points and is incompressible in $\left(S^{3}, K\right)$.

(3) There is a c-weak reduction yielding a torus which intersects $K$ in two or four points and is incompressible in $\left(S^{3}, K\right)$.

Proof Since $\mathcal{S}$ is strongly irreducible and $K$ admits a decomposing sphere, we have the conclusion of Corollary 3.5. Without loss of generality, we assume that there 
are a non-separating cut disk $E_{1}$ of $\mathcal{C}_{1}$ and a compressing disk $E_{2}$ of $\mathcal{C}_{2}$ such that $\partial E_{1} \cap \partial E_{2}=\varnothing$. Then we have:

(i) $E_{2}$ is a non-separating compressing disk of $\mathcal{C}_{2}$,

(ii) $E_{2}$ cuts off a solid torus with two mutually trivial arcs from $\mathcal{C}_{2}$,

(iii) $E_{2}$ cuts off a solid torus with a trivial arc from $\mathcal{C}_{2}$,

(iv) $E_{2}$ cuts off a 3-ball with two mutually trivial arcs from $\mathcal{C}_{2}$, or

(v) $E_{2}$ cuts off a 3 -ball with a single trivial arc from $\mathcal{C}_{2}$.

We do the c-weak reduction with respect to $\left(E_{1}, E_{2}\right)$ to obtain $\mathcal{M}_{i}=\mathcal{C}_{i 1} \cup \mathcal{C}_{i 2}$, $\mathcal{S}_{i}=\mathcal{C}_{i 1} \cap \mathcal{C}_{i 2}$ for each $i=1,2$ and $\mathcal{F}=\partial_{-} \mathcal{C}_{12}=\partial_{-} \mathcal{C}_{21}$.

Case 1 We have the condition (i), (ii) or (iii).

Then there is a non-separating compressing disk of $\mathcal{C}_{2}$ such that its boundary is disjoint from $\partial E_{1}$. Hence it suffices to consider the condition (i) and therefore $\mathcal{F}$ is a 2 -sphere intersecting $K$ in six points. As in the proof of Theorem 5.1, we see that $\mathcal{M}_{1}$ is $\partial$-irreducible. If $\mathcal{M}_{2}$ is also $\partial$-irreducible, then $\mathcal{F}$ is incompressible in $\left(S^{3}, K\right)$ and hence we have the conclusion (2) of Theorem 6.1. Therefore we suppose that $\mathcal{M}_{2}$ is $\partial$-reducible. Then Lemma 2.10 and Corollary 2.11 imply that there is a $\partial$-reducing disk $\bar{D}_{2}$ of $\mathcal{M}_{2}$ with $\bar{D}_{2} \cap E_{1}=\varnothing$. The disk $\bar{D}_{2}$ can be regarded as a compressing disk of $\mathcal{C}_{2}$ which is disjoint from $E_{2}$ and is not parallel to $E_{2}$. Hence $\bar{D}_{2}$ is non-separating in $C_{2}$ (Figure 6 (a), (b) or (c)), $\bar{D}_{2}$ cuts $C_{2}$ into two solid tori (Figure 6 (d) or (e)), or $\bar{D}_{2}$ cuts off a 3 -ball from $C_{2}$ (Figure 6 (f) or $(\mathrm{g})$ ).

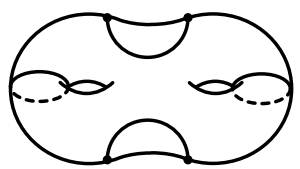

(a)

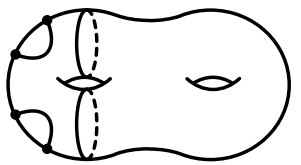

(b)

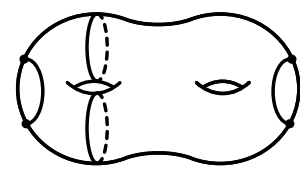

(c)

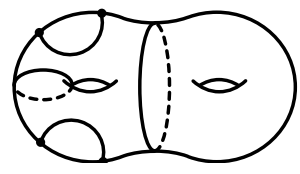

(d)

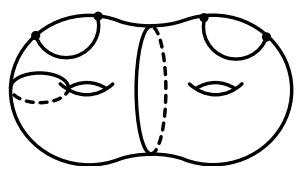

(e)

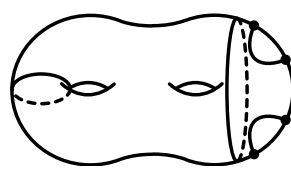

(f)

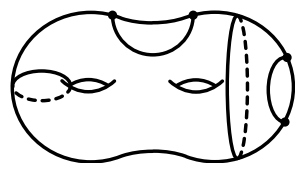

(g)

Figure 6: Possible positions of $\bar{D}_{2} \cup E_{2}$ in $\mathcal{C}_{2}$

If $\bar{D}_{2} \cup E_{2}$ is as illustrated in Figure 6 (a), then $C_{1} \cup C_{2}$ contains a non-separating 2-sphere, a contradiction. If $\bar{D}_{2} \cup E_{2}$ is as illustrated in Figure 6 (b), (d) or (f), then 
we can take $E_{2}$ so that $E_{2}$ satisfies the condition (iv). We will consider this condition in Case 2. If $\bar{D}_{2} \cup E_{2}$ is as illustrated in Figure 6 (c), (e) or (g), then we can take $E_{2}$ so that $E_{2}$ satisfies the condition (v). We will consider this condition in Case 3.

Case 2 We have the condition (iv).
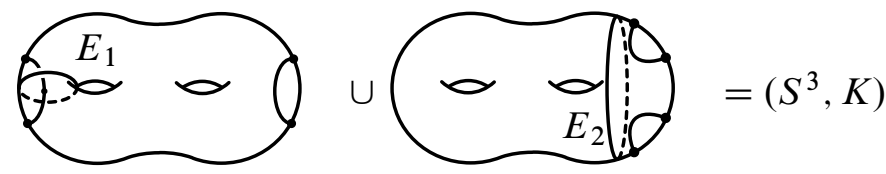

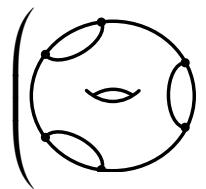

$\mathcal{C}_{11}$

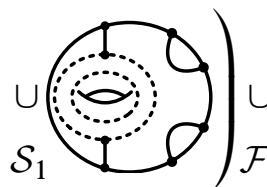

$\mathcal{C}_{12}$

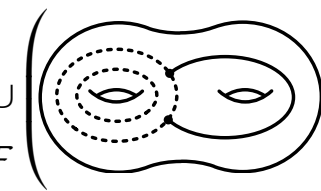

$\mathcal{C}_{21}$

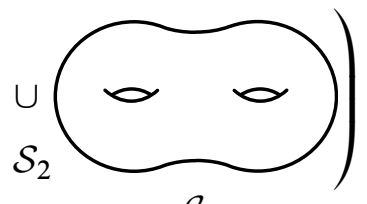

$\mathcal{C}_{22}$

Figure 7: The c-weak reduction with respect to $\left(E_{1}, E_{2}\right)$

Then $\mathcal{F}$ is a torus intersecting $K$ in two points (see Figure 7). If $\mathcal{M}_{1}$ is $\partial$-reducible, then it follows from Corollary 2.11 that $\mathcal{S}_{1}$ is weakly reducible and hence $\mathcal{S}$ is weakly reducible, a contradiction. Hence we see that $\mathcal{M}_{1}$ is $\partial$-irreducible. If $\mathcal{M}_{2}$ is also $\partial$-irreducible, then $\mathcal{F}$ is incompressible in $\left(S^{3}, K\right)$ and hence we have the conclusion (3) of Theorem 6.1. Therefore we suppose that $\mathcal{M}_{2}$ is $\partial$-reducible. Then Lemma 2.10 and Corollary 2.11 imply that there is a $\partial$-reducing disk $\bar{D}_{2}$ of $\mathcal{M}_{2}$ with $\left|\bar{D}_{2} \cap S_{2}\right|=1$ and $\bar{D}_{2} \cap E_{1}=\varnothing$. The disk $\bar{D}_{2}$ can be regarded as a compressing disk of $\mathcal{C}_{2}$ which is disjoint from $E_{2}$ and is not parallel to $E_{2}$. Whether $\bar{D}_{2}$ is separating or non-separating in $C_{2}$, we have the conclusion (1) of Theorem 6.1.

Case 3 We have the condition (v).

Then $\mathcal{F}$ is a torus intersecting $K$ in four points (see Figure 8). As in Case 1, we see that $\mathcal{M}_{1}$ is $\partial$-irreducible. If $\mathcal{M}_{2}$ is also $\partial$-irreducible, then $\mathcal{F}$ is incompressible in $\left(S^{3}, K\right)$ and hence we have the conclusion (3) of Theorem 6.1. Therefore we suppose that $\mathcal{M}_{2}$ is $\partial$-reducible. Then Lemma 2.10 and Corollary 2.11 imply that there is a $\partial$-reducing disk $\bar{D}_{2}$ of $\mathcal{M}_{2}$ with $\left|\bar{D}_{2} \cap S_{2}\right|=1$ and $\bar{D}_{2} \cap E_{1}=\varnothing$. The disk $\bar{D}_{2}$ can be regarded as a compressing disk of $\mathcal{C}_{2}$ which is disjoint from $E_{2}$ and is not parallel to $E_{2}$. Hence there is a non-separating compressing disk $D_{2}$ of $\mathcal{C}_{2}$ with $D_{2} \cap E_{2}=\varnothing$ and $\partial E_{1} \cap \partial\left(D_{2} \cup E_{2}\right)=\varnothing$, or we can retake $E_{2}$ so that $E_{2}$ satisfies the condition 


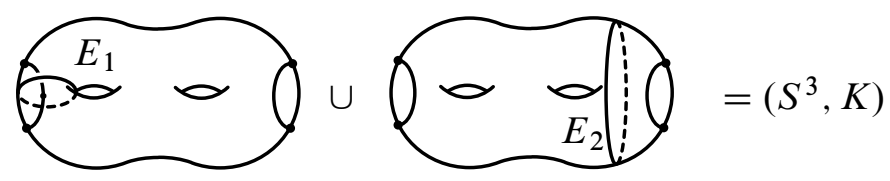

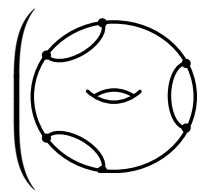

$\mathcal{C}_{11}$

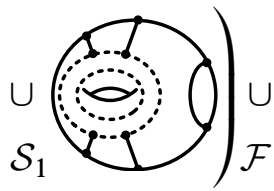

$\mathcal{C}_{12}$
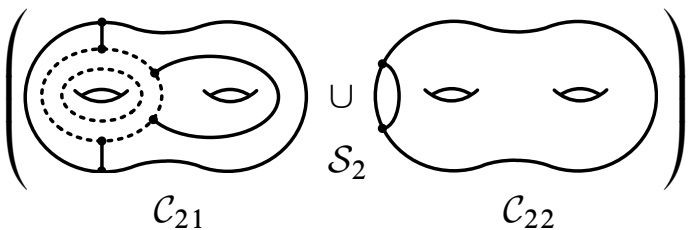

Figure 8: The c-weak reduction with respect to $\left(E_{1}, E_{2}\right)$
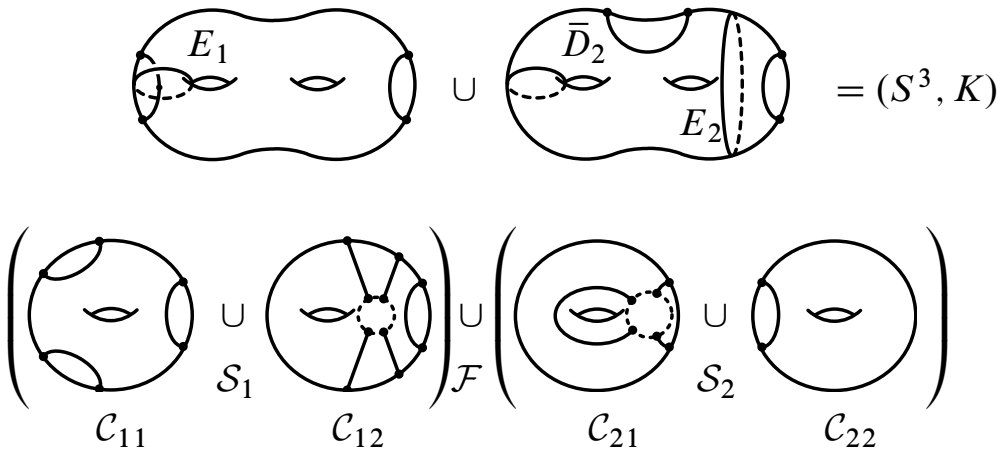

Figure 9: The c-weak reduction with respect to $\left(E_{1}, E_{2}\right)$

(iv). We are done in Case 1 if the latter occurs. Therefore we suppose that the former occurs.

By the c-weak reduction with respect to $\left(E_{1}, D_{2} \cup E_{2}\right)$, we reset $\mathcal{M}_{i}=\mathcal{C}_{i 1} \cup \mathcal{C}_{i 2}$, $\mathcal{S}_{i}=\mathcal{C}_{i 1} \cap \mathcal{C}_{i 2}$ for each $i=1,2$ and $\mathcal{F}=\partial_{-} \mathcal{C}_{12}=\partial_{-} \mathcal{C}_{21}$. We notice that $\mathcal{F}$ is a $2-$ sphere intersecting $K$ in four points (see Figure 9). Since $\mathcal{S}$ is strongly irreducible, we see that $\mathcal{M}_{1}$ is $\partial$-irreducible. If $\mathcal{M}_{2}$ is also $\partial$-irreducible, then $\mathcal{F}$ is incompressible in $\left(S^{3}, K\right)$ and hence we have the conclusion (2) of Theorem 6.1. Therefore we suppose that $\mathcal{M}_{2}$ is $\partial$-reducible. Then Lemma 2.10 and Corollary 2.11 imply that there is a $\partial$-reducing disk $\bar{D}_{2}^{\prime}$ of $\mathcal{M}_{2}$ with $\left|\bar{D}_{2}^{\prime} \cap S_{2}\right|=1$ and $\bar{D}_{2}^{\prime} \cap E_{1}=\varnothing$. The disk $\bar{D}_{2}^{\prime}$ can be regarded as a compressing disk of $\mathcal{C}_{2}$ which is disjoint from $D_{2} \cup E_{2}$ and is parallel neither to $D_{2}$ nor to $E_{2}$. We notice that $\partial E_{1} \cap \partial\left(D_{2} \cup \bar{D}_{2}^{\prime} \cup E_{2}\right)=\varnothing$. Hence we have the conclusion (1) of Theorem 6.1.

This completes the proof of Theorem 6.1. 
Theorem 6.2 Let $K$ be a knot in $S^{3}$ and $\left(\mathcal{C}_{1}, \mathcal{C}_{2} ; \mathcal{S}\right)$ a $(2,2)$-bridge splitting of $\left(S^{3}, K\right)$. Suppose that $\mathcal{S}$ is weakly reducible. Then one of the following holds.

(1) $K=K_{1} \# K_{2}$ such that $K_{1}$ admits a $(0,2)$-bridge position and $K_{2}$ admits a $(2,1)$-bridge position.

(2) $K=K_{1} \# K_{2}$ such that $K_{1}$ admits a $(1,1)$-bridge position and $K_{2}$ admits a $(1,2)$-bridge position.

(3) There is a weak reduction yielding a 2-sphere which intersects $K$ in four points and is incompressible in $\left(S^{3}, K\right)$.

(4) There is a weak reduction yielding a torus which intersects $K$ in two points and is incompressible in $\left(S^{3}, K\right)$.

(5) There is a weak reduction yielding a torus disjoint from $K$ which is incompressible in $\left(S^{3}, K\right)$ and cuts $\left(S^{3}, K\right)$ into the exterior of a tunnel number one knot and a solid torus $V$ with $K$. Moreover, $K$ can be put in a $(1,2)-$ bridge position with respect to a genus one Heegaard surface of $V$.

Proof Let $D_{1}$ and $D_{2}$ be compressing disks of $\mathcal{C}_{1}$ and $\mathcal{C}_{2}$ respectively such that $\partial D_{1} \cap \partial D_{2}=\varnothing$. For each $i=1,2$, we have:

(i) $D_{i}$ is a non-separating compressing disk of $\mathcal{C}_{i}$,

(ii) $D_{i}$ cuts off a solid torus with two mutually trivial arcs from $\mathcal{C}_{i}$,

(iii) $D_{i}$ cuts off a solid torus with a trivial $\operatorname{arc}$ from $\mathcal{C}_{i}$,

(iv) $D_{i}$ cuts off a 3-ball with two mutually trivial $\operatorname{arcs}$ from $\mathcal{C}_{i}$, or

(v) $D_{i}$ cuts off a 3 -ball with a single trivial arc from $\mathcal{C}_{i}$.

We do the weak reduction with respect to $\left(D_{1}, D_{2}\right)$ to obtain $\mathcal{M}_{i}=\mathcal{C}_{i 1} \cup \mathcal{C}_{i 2}, \mathcal{S}_{i}=$ $\mathcal{C}_{i 1} \cap \mathcal{C}_{i 2}$ for each $i=1,2$ and $\mathcal{F}=\partial_{-} \mathcal{C}_{12}=\partial_{-} \mathcal{C}_{21}$.

Case 1 The disk $D_{1}$ satisfies the condition (i), (ii) or (iii).

Suppose first that $D_{2}$ satisfies the condition (i), (ii) or (iii). Then it suffices to consider the case that both $D_{1}$ and $D_{2}$ satisfy the condition (i). Hence $\mathcal{F}$ is a 2-sphere intersecting $K$ in four points. If both $\mathcal{M}_{1}$ and $\mathcal{M}_{2}$ are $\partial$-irreducible, then we have the conclusion (3) of Theorem 6.2. Hence we may assume that $\mathcal{M}_{2}$ is $\partial$-reducible without loss of generality. Then Lemma 2.10 and Corollary 2.11 imply that there is a d-reducing disk $\bar{D}_{2}$ of $\mathcal{M}_{2}$ with $\left|\bar{D}_{2} \cap S_{2}\right|=1$ and $\bar{D}_{2} \cap D_{1}=\varnothing$. The disk $\bar{D}_{2}$ can be regarded as a compressing disk of $\mathcal{C}_{2}$ which is disjoint from $D_{2}$ and is not parallel to $D_{2}$. We notice that $\partial D_{1} \cap \partial\left(D_{2} \cup \bar{D}_{2}\right)=\varnothing$. Since $C_{1} \cup C_{2}$ does not contain a 


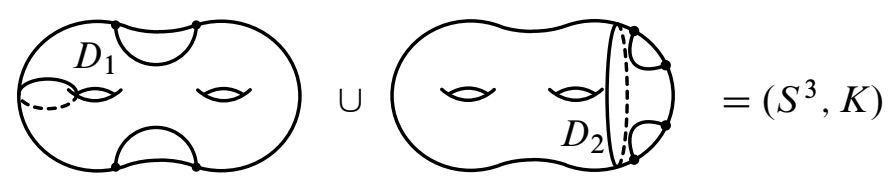

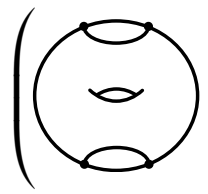

$\mathcal{C}_{11}$

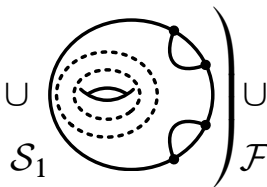

$\mathcal{C}_{12}$

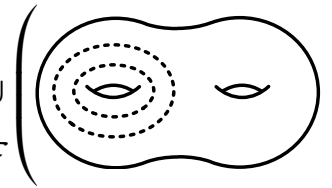

$\mathcal{C}_{21}$

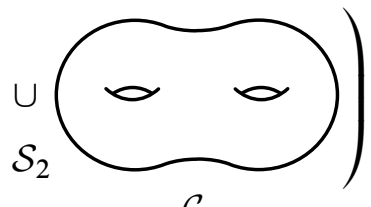

$\mathcal{C}_{22}$

Figure 10: The weak reduction with respect to $\left(D_{1}, D_{2}\right)$

non-separating 2-sphere, we see that $D_{2} \cup \bar{D}_{2}$ is as illustrated in Figure 6 except (a). In each case, we have the conclusion (2) of Theorem 6.2.

Suppose next that $D_{2}$ satisfies the condition (iv). Then $\mathcal{F}$ is a torus disjoint from $K$ (see Figure 10). If both $\mathcal{M}_{1}$ and $\mathcal{M}_{2}$ are $\partial$-irreducible, then we have the conclusion (5) of Theorem 6.2. Hence we assume that $\mathcal{M}_{1}$ or $\mathcal{M}_{2}$ is $\partial$-reducible. If $\mathcal{M}_{1}$ is $\partial$-reducible, then Lemma 2.10 implies that there is a $\partial$-reducing disk $\bar{D}_{1}$ of $\mathcal{M}_{1}$ with $\left|\bar{D}_{1} \cap S_{1}\right|=1$. The disk $\bar{D}_{1}$ can be regarded as a compressing disk of $\mathcal{C}_{1}$ which is disjoint from $D_{1}$ and is not parallel to $D_{1}$. Hence we see that $D_{1} \cup \bar{D}_{1}$ is as illustrated in Figure 6. It follows from Corollary 2.11 that there is a compressing disk $D_{2}^{\prime}$ of $\mathcal{C}_{12}$ with $\bar{D}_{1} \cap D_{2}^{\prime}=\varnothing$. We may assume that $D_{2}^{\prime}$ cuts off a 3 -ball with a trivial arc from $\mathcal{C}_{12}$. Hence the disk $D_{2}^{\prime}$ can be regarded as a compressing disk of $\mathcal{C}_{2}$ with $\partial\left(D_{1} \cup \bar{D}_{1}\right) \cap \partial D_{2}^{\prime}=\varnothing$. Therefore we have the conclusion (1) of Theorem 6.2. If $\mathcal{M}_{2}$ is $\partial$-reducible, then $\mathcal{M}_{2}$ is a solid torus. This implies that $K$ admits a $(1,2)$-bridge position and therefore we have the conclusion (2) of Theorem 6.2.

Suppose finally that $D_{2}$ satisfies the condition (v) (see Figure 11). If both $\mathcal{M}_{1}$ and $\mathcal{M}_{2}$ are $\partial$-irreducible, then we have the conclusion (4) of Theorem 6.2. If $\mathcal{M}_{1}$ is $\partial$-reducible, then we have the conclusion (1) of Theorem 6.2 by an argument similar to the above. If $\mathcal{M}_{2}$ is $\partial$-reducible, then we have the conclusion (2) of Theorem 6.2, or we can retake $D_{2}$ so that $\partial D_{1} \cap \partial D_{2}=\varnothing$ and $D_{2}$ satisfies the condition (iv) and hence we are done.

Case 2 The disk $D_{1}$ satisfies the condition (iv) or (v).

Suppose that $D_{1}$ satisfies the condition (iv). Then we have the conclusion (1) of Theorem 6.2, whether $D_{2}$ satisfies the condition (iv) or (v). It is impossible to have the condition $(\mathrm{v})$, because $K$ is connected. 


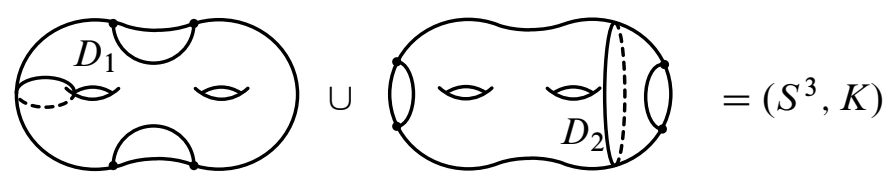

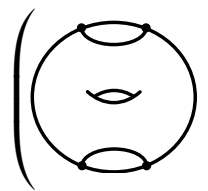

$\mathcal{C}_{11}$

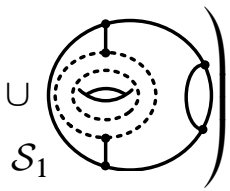

$\mathcal{C}_{12}$

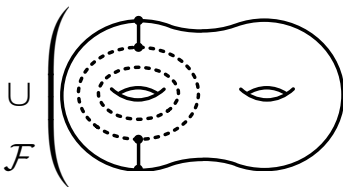

$\mathcal{C}_{21}$

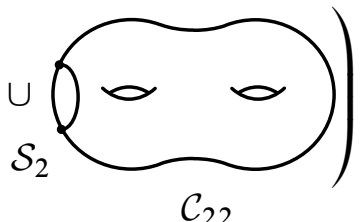

$\mathcal{C}_{22}$

Figure 11: The weak reduction with respect to $\left(D_{1}, D_{2}\right)$

This completes the proof of Theorem 6.2.

\section{The connected sum of $n$-string prime knots}

Proposition 7.1 For each $i=1,2$, let $K_{i}$ be a non-trivial knot in $S^{3}$ and set $K=$ $K_{1} \# K_{2}$. Suppose that $\left(S^{3}, K\right)$ admits a closed incompressible surface $\mathcal{F}=(F, F \cap K)$ with $F \cap K \neq \varnothing$ and $\chi\left(F \cap \operatorname{Ext}\left(K ; S^{3}\right)\right)<0$, where $\chi(\cdot)$ is the Euler characteristic. Then for $i=1$ or $2,\left(S^{3}, K_{i}\right)$ admits a closed surface $\mathcal{F}^{\prime}=\left(F^{\prime}, F^{\prime} \cap K_{i}\right)$, which is obtained from a subsurface of $F$ by filling its boundary with disjoint disks, such that $\mathcal{F}^{\prime}$ is incompressible in $\left(S^{3}, K_{i}\right), F^{\prime} \cap K_{i} \neq \varnothing$ and $\chi\left(F^{\prime} \cap \operatorname{Ext}\left(K_{i} ; S^{3}\right)\right)<0$.

Proof Let $\Sigma$ be a decomposing sphere of $K$ with $K=K_{1} \#_{\Sigma} K_{2}$. Then $\Sigma$ divides $\left(S^{3}, K\right)$ into $\mathcal{B}_{1}=\left(B_{1}, K_{1}^{\prime}\right)$ and $\mathcal{B}_{2}=\left(B_{2}, K_{2}^{\prime}\right)$, where $B_{i}$ is a 3 -ball and $K_{i}^{\prime}=$ $K \cap B_{i}(i=1,2)$. We notice that $\left(B_{1}, K_{1}^{\prime}\right)$ forms $\left(S^{3}, K_{1}\right)$ after gluing a 3 -ball $B$ with a trivial arc $\gamma$. We set $\mathcal{B}=(B, \gamma)$. If $F \cap \Sigma=\varnothing$, then we are done. Hence we assume that $F \cap \Sigma \neq \varnothing$. It follows from Lemma 3.1 that $F$ and $\Sigma$ are isotoped so that each component of $F \cap \Sigma$ is non-trivial both in $\mathcal{F}$ and in $\Sigma$. Since $\chi\left(F \cap \operatorname{Ext}\left(K ; S^{3}\right)\right)<0$, there is a component $F_{0}$ of $F$ cut along $F \cap \Sigma$ such that $\chi\left(F_{0} \cap \operatorname{Ext}\left(K ; S^{3}\right)\right)<0$. Without loss of generality, we may assume that $F_{0} \subset B_{1}$. Recall that $\left(S^{3}, K_{1}\right)=\mathcal{B}_{1} \cup \mathcal{B}$. Let $F^{\prime}$ be a closed surface obtained from $F_{0}$ by filling its boundary with disjoint disks in $B$ such that each disk intersects the trivial arc $\gamma$ in a single point. Then $F^{\prime}$ is a closed surface in $S^{3}=B_{1} \cup B$. Set $\mathcal{F}^{\prime}=\left(F^{\prime}, F^{\prime} \cap K_{1}\right)$. Suppose that $\mathcal{F}^{\prime}$ is compressible in $\left(S^{3}, K_{1}\right)$, and let $\delta$ be its compressing disk. We may assume that $\partial \delta$ is contained in $F_{0} \subset F^{\prime}$ and moreover $\delta \subset B_{1}$. By an innermost disk argument, if necessary, we see that $\mathcal{F}$ is compressible in $\left(S^{3}, K\right)$, a contradiction. Hence $\mathcal{F}^{\prime}$ is incompressible in $\left(S^{3}, K_{1}\right)$. 
A knot in $S^{3}$ is said to be $n$-string prime $(n>0)$ if there is no incompressible 2 -sphere intersecting the knot in $2 n$ points.

Corollary 7.2 Let $K$ be the connected sum of non-trivial knots of $n$-string prime for all $n$. Then $\left(S^{3}, K\right)$ admits no incompressible 2 -spheres intersecting $K$ in more than two points.

Proposition 7.3 Let $K$ be the connected sum of non-trivial knots of $n$-string prime for all $n$. Suppose that $\left(S^{3}, K\right)$ admits an incompressible torus $\mathcal{T}=(T, T \cap K)$ which is not isotopic to $\partial \eta\left(K ; S^{3}\right)$. Then there is a decomposing sphere of $K$ disjoint from $T$.

Proof Let $\Sigma$ be a decomposing sphere of $K$. If $\Sigma \cap T=\varnothing$, then we are done. Hence we assume that $\Sigma \cap T \neq \varnothing$. Then it follows from Lemma 3.1 that $\Sigma$ and $T$ are isotoped so that each component of $\Sigma \cap T$ is non-trivial both in $\Sigma$ and in $\mathcal{T}$. We take $\Sigma$ so that $|\Sigma \cap T|$ is minimal among such all decomposing spheres of $K$.

We first suppose that $T \cap K=\varnothing$. Then $T$ cuts off a pair of a solid torus $V$ and the knot $K$ from $\left(S^{3}, K\right)$. Let $\Delta$ be a disk component of $\Sigma$ cut along $\Sigma \cap T$. We notice that $\Delta$ intersects $K$ in a single point and hence $\Delta$ is a cut disk of $(V, K)$. Let $\Sigma^{\prime}$ be a $2-$ sphere obtained by cutting $T$ along $\partial \Delta$ and attaching copies of $\Delta$ to the resulting boundaries. Since $K$ is not a core loop of $V$, we see that $\Sigma^{\prime}$ bounds a 3-ball $B$ in $V$ which contains a non-trivial arc. Since $T$ is incompressible in $\left(S^{3}, K\right)$, we see that $\operatorname{Ext}\left(V ; S^{3}\right)$ is not a solid torus and therefore $\operatorname{Ext}\left(B ; S^{3}\right)$ is a 3 -ball which contains a non-trivial arc. Hence $\Sigma^{\prime}$ is a decomposing sphere of $K$ disjoint from $T$. This contradicts the minimality of $|\Sigma \cap T|$.

We now suppose that $T \cap K \neq \varnothing$. If a component of $\Sigma \cap T$ is essential in $\mathcal{T}$, then there is a component $T_{0}$ of $T$ cut along $\Sigma \cap T$ such that $T_{0} \cap E\left(K ; S^{3}\right)$ is a planar surface with $\chi\left(T_{0} \cap \operatorname{Ext}\left(K ; S^{3}\right)\right)<0$. This together with Proposition 7.1 implies that for a factor knot $K^{\prime}$ of $K,\left(S^{3}, K^{\prime}\right)$ admits an incompressible 2-sphere $\mathcal{P}=\left(P, P \cap K^{\prime}\right)$ with $P \cap K^{\prime} \neq \varnothing$ and $\chi\left(P \cap \operatorname{Ext}\left(K^{\prime} ; S^{3}\right)\right)<0$. This contradicts that $K$ is the connected sum of non-trivial knots of $n$-string prime for all $n$. Hence each component of $\Sigma \cap T$ bounds a disk in $T$ which intersects $K$ in a single point. Let $\alpha$ be a component of $\Sigma \cap T$ which is innermost in $T$ and $\delta_{\alpha}$ its innermost disk. Since each component of $\Sigma \cap T$ is non-trivial in $\Sigma, \alpha$ bounds a disk $\delta_{\alpha}^{\prime}$ in $\Sigma$ intersecting $K$ in a single point. If $\Sigma^{\prime}=\delta_{\alpha} \cup \delta_{\alpha}^{\prime}$ bounds a 3-ball with a trivial arc, then we can isotope $\Sigma$ and $T$ so that $|\Sigma \cap T|$ is reduced, a contradiction. Therefore $\Sigma^{\prime}$ is a decomposing sphere of $K$. A slight isotopy implies that $\left|\Sigma^{\prime} \cap T\right|<|\Sigma \cap T|$. This also contradicts the minimality of $|\Sigma \cap T|$. 
Let $K$ be the connected sum of non-trivial knots of $n$-string prime for all $n$, and let $\left(\mathcal{C}_{1}, \mathcal{C}_{2} ; \mathcal{S}\right)$ be a $(1,3)$ - or $(2,2)$-bridge splitting of $\left(S^{3}, K\right)$. Then it follows from Corollary 7.2 that $\left(S^{3}, K\right)$ admits no incompressible 2 -spheres intersecting $K$ in more than two points. Toward Theorem 1.4, we need to study up on all the cases such that an incompressible torus is obtained by a weak or c-weak reduction in Sections 5 and 6. Namely, $\left(\mathcal{C}_{1}, \mathcal{C}_{2} ; \mathcal{S}\right)$ is one of the following:

(I) a $(1,3)$-bridge splitting as illustrated in Figure 5,

(II) a $(2,2)$-bridge splitting as illustrated in Figure 7,

(III) a $(2,2)$-bridge splitting as illustrated in Figure 8,

(IV) a $(2,2)$-bridge splitting as illustrated in Figure 10, and

(V) a $(2,2)$-bridge splitting as illustrated in Figure 11.

Theorem 7.4 Let $K$ be the connected sum of non-trivial knots of $n$-string prime for all $n$ and $\left(\mathcal{C}_{1}, \mathcal{C}_{2} ; \mathcal{S}\right)$ a $(1,3)$-bridge splitting of $\left(S^{3}, K\right)$. Suppose that there are compressing disks $D_{1}$ and $D_{2}$ of $\mathcal{C}_{1}$ and $\mathcal{C}_{2}$ respectively such that $\partial D_{1} \cap \partial D_{2}=\varnothing$ and that each $D_{i}$ cuts off a 3-ball with a single trivial arc from $\mathcal{C}_{i}$. Then a torus obtained by the weak reduction with respect to $\left(D_{1}, D_{2}\right)$ is compressible in $\left(S^{3}, K\right)$.

Proof As in Figure 5, we obtain $\mathcal{M}_{i}=\mathcal{C}_{i 1} \cup \mathcal{C}_{i 2}, \mathcal{S}_{i}=\mathcal{C}_{i 1} \cap \mathcal{C}_{i 2}$ for each $i=1,2$ and $\mathcal{F}=\partial_{-} \mathcal{C}_{12}=\partial_{-} \mathcal{C}_{21}$ by the weak reduction with respect to $\left(D_{1}, D_{2}\right)$. We notice that $\mathcal{F}=(F, F \cap K)$ is a torus. Suppose, towards a contradiction, that $\mathcal{F}=(F, F \cap K)$ is incompressible in $\left(S^{3}, K\right)$.

Claim The surface $\mathcal{S}_{i}=\left(S_{i}, S_{i} \cap K\right)$ is strongly irreducible for each $i=1,2$.

Proof Suppose that $\mathcal{S}_{i}$, say $i=1$, is weakly reducible. We notice that a compressing disk of $\mathcal{C}_{12}$ is isotopic to $\hat{D}_{2}$, where $\hat{D}_{2}$ is a compressing disk of $\mathcal{C}_{12}$ which is obtained by extending $D_{2}$ naturally. Hence there is a compressing disk $D_{11}$ of $\mathcal{C}_{11}$ with $\partial D_{11} \cap \partial \widehat{D}_{2}=\varnothing$. We notice that $\mathcal{S}_{1}$ is irreducible because $K$ is connected. This implies that there is a vertical annulus $A_{12}$ in $C_{12}$ with $A_{12} \cap K=\varnothing, A_{12} \cap \widehat{D}_{2}=\varnothing$ and $\partial A_{12} \supset \partial D_{11}$. Hence $D_{11} \cup A_{12}$ is a $\partial$-reducing disk of $\mathcal{M}_{1}$ and therefore $\mathcal{F}$ is compressible, contrary to the hypothesis.

It follows from Proposition 7.3 that there is a decomposing sphere of $K$ disjoint from $F$. Without loss of generality, we may assume that a decomposing sphere is contained in $\mathcal{M}_{1}$. Then the $\mathrm{cH}$-splitting $\left(\mathcal{C}_{11}, \mathcal{C}_{12} ; \mathcal{S}_{1}\right)$ satisfies one of the conclusions of Corollary 3.4. Moreover, we see that the conclusion (1) of Corollary 3.4 does not hold by an argument similar to that in the proof of Corollary 3.5. Hence we have only the conclusion (2) of Corollary 3.4. 
Case 1 There are a cut disk $E_{11}$ of $\mathcal{C}_{11}$ and a compressing disk $E_{12}$ of $\mathcal{C}_{12}$ such that $\partial E_{11} \cap \partial E_{12}=\varnothing$.
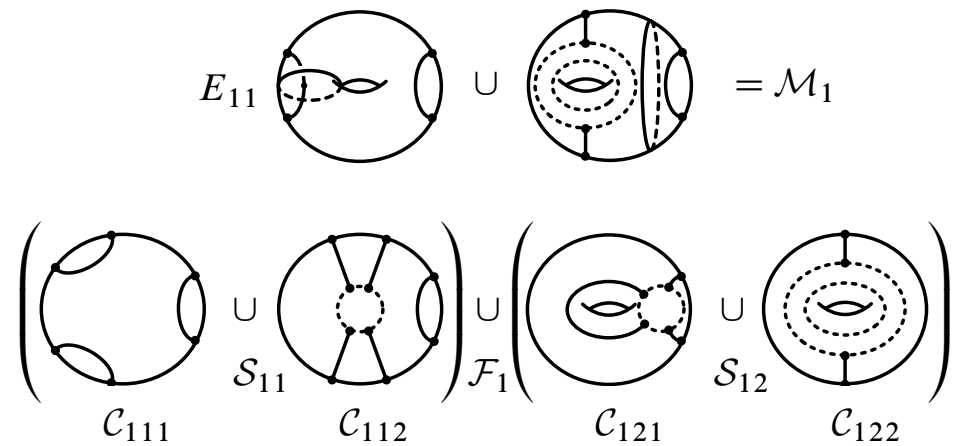

Figure 12: The c-weak reduction with respect to $\left(E_{11}, \widehat{D}_{2}\right)$

Then we see that $E_{11}$ is non-separating $C_{11}$ by an argument similar to that in the proof of Corollary 3.5. We notice that $E_{12}$ is isotopic to $\hat{D}_{2}$ by Lemma 2.4. We now do the c-weak reduction with respect to $\left(E_{11}, \hat{D}_{2}\right)$. As usual, we set $\mathcal{M}_{1 i}=\mathcal{C}_{1 i 1} \cup \mathcal{C}_{1 i 2}$, $\mathcal{S}_{1 i}=\mathcal{C}_{1 i 1} \cap \mathcal{C}_{1 i 2}$ for each $i=1,2$ and $\mathcal{F}_{1}=\partial_{-} \mathcal{C}_{112}=\partial_{-} \mathcal{C}_{121}$ (see Figure 12 ). We notice that $\mathcal{F}_{1}$ is a 2 -sphere intersecting $K$ in four points. Since we assume that $K$ is the connected sum of non-trivial knots of $n$-string prime for all $n$, it follows from Corollary 7.2 that $\mathcal{F}_{1}$ is compressible in $\left(S^{3}, K\right)$. Let $D$ be a compressing disk of $\mathcal{F}_{1}$. Since $\mathcal{F}$ is incompressible in $\left(S^{3}, K\right)$, we may assume that $D$ is disjoint from $F$. This implies that $\mathcal{F}_{1}$ is compressible in $\mathcal{M}_{1}$ and hence either $\mathcal{M}_{11}$ or $\mathcal{M}_{12}$ is $\partial$-reducible. If $\mathcal{M}_{11}$ is $\partial$-reducible, then we see that $\mathcal{S}_{1}$ is weakly reducible by Corollary 2.11 . This contradicts the claim above. We also see that $\mathcal{M}_{12}$ is $\partial$-irreducible because $C_{12 j}$ is ambient isotopic to a regular neighborhood of $\partial_{-} C_{12 j} \cup\left(C_{12 j} \cup K\right)$ for each $j=1,2$. Therefore Case 1 does not hold.

Case 2 There are a compressing disk $E_{11}$ of $\mathcal{C}_{11}$ and a cut disk $E_{12}$ of $\mathcal{C}_{12}$ such that $\partial E_{11} \cap \partial E_{12}=\varnothing$.

Since $C_{12}$ is homeomorphic to $\partial_{-} C_{12} \times[0,1]$, we see that $E_{12}$ cuts off a 3 -ball $B$ from $C_{12}$. Since $\partial E_{12}$ is essential in $\mathcal{S}_{1}$, we see that $E_{12}$ intersects a vertical arc of $C_{12} \cap K$ and hence $B$ intersects $K$ in two mutually trivial arcs one of which is the trivial arc in $\mathcal{C}_{12}$. Namely, $E_{12}$ cuts $\mathcal{C}_{12}$ into a 3 -manifold $\partial_{-} C_{12} \times[0,1]$ with two vertical arcs and the 3 -ball $B$ with two trivial arcs. If $E_{11}$ is non-separating in $C_{11}$, then $\partial E_{11}$ is disjoint from $B$. This implies that $\mathcal{S}_{1}$ is weakly reducible, a contradiction. 
Hence $E_{11}$ cuts off a 3 -ball with one or two trivial arcs from $\mathcal{C}_{11}$. However, this also implies that $\mathcal{S}_{1}$ is weakly reducible, a contradiction.

This completes the proof of Theorem 7.4.

Theorem 7.5 Let $K$ be the connected sum of non-trivial knots of $n$-string prime for all $n$ and $\left(\mathcal{C}_{1}, \mathcal{C}_{2} ; \mathcal{S}\right)$ a strongly irreducible $(2,2)$-bridge splitting of $\left(S^{3}, K\right)$. Suppose that there are a non-separating cut disk $E_{1}$ of $\mathcal{C}_{1}$ and a compressing disk $E_{2}$ of $\mathcal{C}_{2}$ such that $\partial E_{1} \cap \partial E_{2}=\varnothing$ and that $E_{2}$ cuts off a 3-ball with two mutually trivial arcs from $\mathcal{C}_{2}$. Then a torus obtained by the c-weak reduction with respect to $\left(E_{1}, E_{2}\right)$ is compressible in $\left(S^{3}, K\right)$.

Proof Suppose, towards a contradiction, that a torus obtained by the c-weak reduction with respect to $\left(E_{1}, E_{2}\right)$ is incompressible in $\left(S^{3}, K\right)$. As in the proof of Theorem 6.1, we obtain $\mathcal{M}_{i}=\mathcal{C}_{i 1} \cup \mathcal{C}_{i 2}, \mathcal{S}_{i}=\mathcal{C}_{i 1} \cap \mathcal{C}_{i 2}$ for each $i=1,2$ and $\mathcal{F}=\partial_{-} \mathcal{C}_{12}=$ $\partial_{-} \mathcal{C}_{21}$ by the c-weak reduction with respect to $\left(E_{1}, E_{2}\right)$ (see Figure 7 ). Recall that $\mathcal{F}=(F, F \cap K)$ is a torus of which we suppose incompressibility. It follows from Proposition 7.3 that there is a decomposing sphere of $K$ disjoint from $F$. Suppose that a decomposing sphere is contained in $\mathcal{M}_{2}$. We notice that $\mathcal{S}_{2}$ is strongly irreducible because $C_{21}$ is ambient isotopic to a regular neighborhood of $\partial_{-} C_{21} \cup\left(C_{21} \cap K\right)$. Hence the $\mathrm{cH}$-splitting $\left(\mathcal{C}_{21}, \mathcal{C}_{22} ; \mathcal{S}_{2}\right)$ satisfies one of the conclusions of Corollary 3.4. Moreover, we see that the conclusion (1) of Corollary 3.4 does not hold because $\mathcal{C}_{21}$ admits no separating cut disks. Hence we have only the conclusion (2) of Corollary 3.4. This implies that there are a cut disk $E_{21}$ of $\mathcal{C}_{21}$ and a compressing disk $E_{22}$ of $\mathcal{C}_{22}$ such that $\partial E_{21} \cap \partial E_{22}=\varnothing$. Then we can extend $E_{22}$ into $C_{1}$ so that the extended disk is a compressing disk of $\mathcal{F}$, a contradiction. Therefore a decomposing sphere is contained in $\mathcal{M}_{1}$.

Claim There are a non-separating cut disk $E_{11}$ of $\mathcal{C}_{11}$ and a compressing disk $E_{12}$ of $\mathcal{C}_{12}$ with $\partial E_{11} \cap \partial E_{12}=\varnothing$.

Proof If $\mathcal{S}_{1}$ is weakly reducible, then $\mathcal{S}$ is also weakly reducible. Hence $\mathcal{S}_{1}$ is strongly irreducible. Hence the $\mathrm{cH}$-splitting $\left(\mathcal{C}_{11}, \mathcal{C}_{12} ; \mathcal{S}_{1}\right)$ satisfies one of the conclusions of Corollary 3.4. Moreover, we have only the conclusion (2) of Corollary 3.4. If there are a compressing disk $E_{11}$ of $\mathcal{C}_{11}$ and a cut disk $E_{12}$ of $\mathcal{C}_{12}$ such that $\partial E_{11} \cap \partial E_{12}=\varnothing$, then either $\mathcal{S}_{1}$ is weakly reducible, or $\mathcal{F}$ is compressible. Hence there are a cut disk $E_{11}$ of $\mathcal{C}_{11}$ and a compressing disk $E_{12}$ of $\mathcal{C}_{12}$ such that $\partial E_{11} \cap \partial E_{12}=\varnothing$. By an argument similar to that in the proof of Corollary 3.5, we see that $E_{11}$ must be non-separating in $C_{11}$ because $\mathcal{S}_{1}$ is strongly irreducible. 


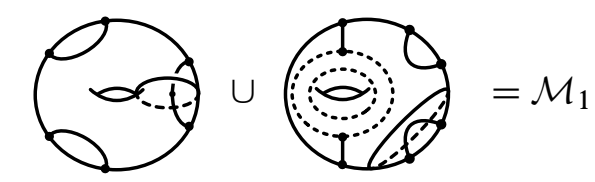

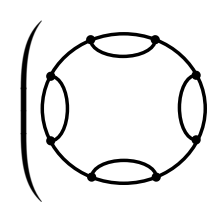

$\mathcal{C}_{111}$

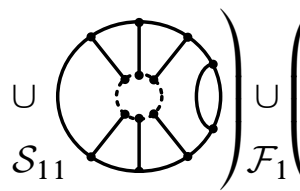

$\mathcal{C}_{112}$

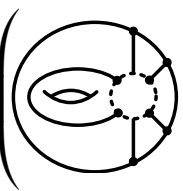

$\mathcal{C}_{121}$

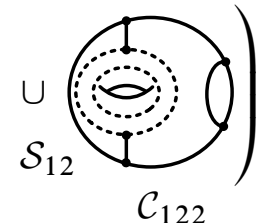

$\mathcal{C}_{122}$

Figure 13: The c-weak reduction with respect to $\left(E_{11}, E_{12}\right)$

Case 1 The disk $E_{12}$ cuts off a 3 -ball with a trivial arc from $\mathcal{C}_{12}$.

We now do the c-weak reduction with respect to $\left(E_{11}, E_{12}\right)$. As usual, we set $\mathcal{M}_{1 i}=$ $\mathcal{C}_{1 i 1} \cup \mathcal{C}_{1 i 2}, \mathcal{S}_{1 i}=\mathcal{C}_{1 i 1} \cap \mathcal{C}_{1 i 2}$ for each $i=1,2$ and $\mathcal{F}_{1}=\partial_{-} \mathcal{C}_{112}=\partial_{-} \mathcal{C}_{121}$ (see Figure 13). We notice that $\mathcal{F}_{1}$ is a 2 -sphere intersecting $K$ in six points. Since we assume that $K$ is the connected sum of non-trivial knots of $n$-string prime for all $n$, it follows from Proposition 7.1 that $\mathcal{F}_{1}$ is compressible in $\left(S^{3}, K\right)$. Let $D$ be a compressing disk of $\mathcal{F}_{1}$. Since $\mathcal{F}$ is incompressible in $\left(S^{3}, K\right)$, we may assume that $D$ is disjoint from $F$. This implies that $\mathcal{F}_{1}$ is compressible in $\mathcal{M}_{1}$ and hence either $\mathcal{M}_{11}$ or $\mathcal{M}_{12}$ is $\partial$-reducible. If $\mathcal{M}_{11}$ is $\partial$-reducible, then $\mathcal{S}_{11}$ is weakly reducible and hence $\mathcal{S}_{1}$ is weakly reducible, a contradiction. Hence $\mathcal{M}_{12}$ is $\partial$-reducible and therefore there is a compressing disk $D_{122}$ of $\mathcal{C}_{122}$ such that its boundary is disjoint from $\partial E_{11}$. We can regard $\partial D_{122}$ as a compressing disk of $\mathcal{C}_{12}$ which is disjoint from $E_{12}$ and is not parallel to $E_{12}$. We notice that $\partial E_{11} \cap \partial\left(D_{122} \cup E_{12}\right)=\varnothing$. This implies that there is a compressing disk of $\mathcal{C}_{12}$, which is obtained by joining $D_{122}$ to $E_{12}$ with a band, such that its boundary is disjoint from $\partial E_{11}$ and that it cuts off a 3 -ball with two mutually trivial arcs from $\mathcal{C}_{12}$. We consider such a case in the following.

Case 2 The disk $E_{12}$ cuts off a 3 -ball with two mutually trivial arcs from $\mathcal{C}_{12}$.

We now do the c-weak reduction with respect to $\left(E_{11}, E_{12}\right)$. As usual, we set $\mathcal{M}_{1 i}=$ $\mathcal{C}_{1 i 1} \cup \mathcal{C}_{1 i 2}, \mathcal{S}_{1 i}=\mathcal{C}_{1 i 1} \cap \mathcal{C}_{1 i 2}$ for each $i=1,2$ and $\mathcal{F}_{1}=\partial_{-} \mathcal{C}_{112}=\partial_{-} \mathcal{C}_{121}$. We notice that $\mathcal{F}_{1}$ is a 2 -sphere intersecting $K$ in four points. If $\mathcal{M}_{11}$ is $\partial$-reducible, then we see that $\mathcal{S}_{1}$ is weakly reducible, a contradiction. Since $C_{12 i}$ is ambient isotopic to a regular neighborhood of $\partial_{-} C_{12 i} \cup\left(C_{12 i} \cup K\right)$ for each $i=1,2$, we see that $\mathcal{M}_{12}$ is 
also $\partial$-irreducible. This implies that $\mathcal{F}_{1}$ is incompressible in $\left(S^{3}, K\right)$, contradicting that $K$ is the connected sum of non-trivial knots of $n$-string prime for all $n$.

This completes the proof of Theorem 7.5.

Theorem 7.6 Let $K$ be the connected sum of non-trivial knots of $n$-string prime for all $n$ and $\left(\mathcal{C}_{1}, \mathcal{C}_{2} ; \mathcal{S}\right)$ a strongly irreducible $(2,2)$-bridge splitting of $\left(S^{3}, K\right)$. Suppose that there are a non-separating cut disk $E_{1}$ and a compressing disk $E_{2}$ of $\mathcal{C}_{1}$ and $\mathcal{C}_{2}$ respectively such that $\partial E_{1} \cap \partial E_{2}=\varnothing$ and that $E_{2}$ cuts off a 3-ball with a trivial arc from $\mathcal{C}_{2}$. Then a torus obtained by the c-weak reduction with respect to $\left(E_{1}, E_{2}\right)$ is compressible in $\left(S^{3}, K\right)$.

Proof Suppose, towards a contradiction, that a torus obtained by the c-weak reduction with respect to $\left(E_{1}, E_{2}\right)$ is incompressible in $\left(S^{3}, K\right)$. As in the proof of Theorem 6.1, we obtain $\mathcal{M}_{i}=\mathcal{C}_{i 1} \cup \mathcal{C}_{i 2}, \mathcal{S}_{i}=\mathcal{C}_{i 1} \cap \mathcal{C}_{i 2}$ for each $i=1,2$ and $\mathcal{F}=\partial_{-} \mathcal{C}_{12}=$ $\partial_{-} \mathcal{C}_{21}$ by the c-weak reduction with respect to $\left(E_{1}, E_{2}\right)$ (see Figure 8 ). Recall that $\mathcal{F}=(F, F \cap K)$ is a torus of which we suppose incompressibility. It follows from Proposition 7.3 that there is a decomposing sphere of $K$ disjoint from $F$. By an argument similar to that in the first half of the proof of Theorem 7.5, we see that a decomposing sphere is contained in $\mathcal{M}_{1}$. The argument to obtain the desired result is almost the same as that in the proof of Theorem 7.4.

Theorem 7.7 Let $K$ be the connected sum of non-trivial knots of $n$-string prime for all $n$ and $\left(\mathcal{C}_{1}, \mathcal{C}_{2} ; \mathcal{S}\right)$ a $(2,2)$-bridge splitting of $\left(S^{3}, K\right)$. Suppose that there are compressing disks $D_{1}$ and $D_{2}$ of $\mathcal{C}_{1}$ and $\mathcal{C}_{2}$ respectively such that $\partial D_{1} \cap \partial D_{2}=\varnothing$, $D_{1}$ is non-separating, and that $D_{2}$ cuts off a 3-ball with two mutually trivial arcs from $\mathcal{C}_{2}$. Suppose also that a torus obtained by the weak reduction with respect to $\left(D_{1}, D_{2}\right)$ is incompressible in $\left(S^{3}, K\right)$. Then one of the following holds.

(1) $K$ contains a non-trivial 2-bridge knot as a connected summand.

(2) $K=K_{1} \# K_{2}$ such that each $K_{i}$ admits a $(1,1)$-bridge position.

(3) $K=K_{1} \# K_{2}$ such that $K_{1}$ admits a $(0,3)$-bridge position and $K_{2}$ admits a $(2,0)$-bridge position.

Proof As in the proof of Theorem 6.2, we obtain $\mathcal{M}_{i}=\mathcal{C}_{i 1} \cup \mathcal{C}_{i 2}, \mathcal{S}_{i}=\mathcal{C}_{i 1} \cap \mathcal{C}_{i 2}$ for each $i=1,2$ and $\mathcal{F}=\partial_{-} \mathcal{C}_{12}=\partial_{-} \mathcal{C}_{21}$ by the weak reduction with respect to $\left(D_{1}, D_{2}\right)$ (see Figure 10). Recall that $\mathcal{F}=(F, F \cap K)$ is a torus of which we suppose incompressibility. It follows from Proposition 7.3 that there is a decomposing sphere of $K$ disjoint from $F$. Hence $\mathcal{M}_{1}$ contains a decomposing sphere of $K$. Suppose that $\mathcal{S}_{1}$ is weakly reducible. Then there are compressing disks $D_{11}$ and $D_{12}$ of $\mathcal{C}_{11}$ 
and $\mathcal{C}_{12}$ respectively such that $\partial D_{11} \cap \partial D_{12}=\varnothing$. We may assume that $D_{11}$ is nonseparating in $C_{11}$ and that $D_{12}$ cuts off a 3-ball with a trivial arc from $\mathcal{C}_{12}$. Then $K$ contains a non-trivial 2-bridge knot as a connected summand, or $\left(\mathcal{C}_{1}, \mathcal{C}_{2} ; \mathcal{S}\right)$ is simplified so that $\left(S^{3}, K\right)$ admits a $(2,1)$-bridge decomposition. If the latter occurs, then it follows from Morimoto [13, Theorem 1.6] that we have the conclusion (1) or (2) of Theorem 7.7. Therefore we assume that $\mathcal{S}_{1}$ is strongly irreducible. This implies that there are a non-separating cut disk $E_{11}$ of $\mathcal{C}_{11}$ and a compressing disk $E_{12}$ of $\mathcal{C}_{12}$ with $\partial E_{11} \cap \partial E_{12}=\varnothing$ (see Corollary 3.5).

The following argument is quite similar to that in the proof of Theorem 7.5. We now do the c-weak reduction with respect to $\left(E_{11}, E_{12}\right)$. As usual we set $\mathcal{M}_{1 i}=\mathcal{C}_{1 i 1} \cup \mathcal{C}_{1 i 2}$, $\mathcal{S}_{1 i}=\mathcal{C}_{1 i 1} \cap \mathcal{C}_{1 i 2}$ for each $i=1,2$ and $\mathcal{F}_{1}=\partial_{-} \mathcal{C}_{112}=\partial_{-} \mathcal{C}_{121}$. We suppose that $E_{12}$ cuts off a 3 -ball with a trivial arc from $\mathcal{C}_{12}$. Then $\mathcal{F}_{1}$ is a 2 -sphere intersecting $K$ in four points. Since we assume that $K$ is the connected sum of non-trivial knots of $n$-string prime for all $n$, we see that $\mathcal{F}_{1}$ is compressible in $\mathcal{M}_{1}$ and hence either $\mathcal{M}_{11}$ or $\mathcal{M}_{12}$ is $\partial$-reducible. If $\mathcal{M}_{11}$ is $\partial$-reducible, then $\mathcal{S}_{1}$ is weakly reducible, a contradiction. Hence $\mathcal{M}_{12}$ is $\partial$-reducible. Since $C_{121}$ is isotopic to a regular neighborhood of $\partial_{-} C_{121} \cup\left(C_{121} \cap K\right)$, there is a $\partial$-reducing disk $\bar{D}_{12}$ of $\mathcal{M}_{12}$ with $\left|\bar{D}_{12} \cap \mathcal{S}_{12}\right|=1$ and $\bar{D}_{12} \cap E_{11}=\varnothing$. We can regard $\bar{D}_{12}$ as a compressing disk of $\mathcal{C}_{12}$ which is disjoint from $E_{12}$ and is not parallel to $E_{12}$. This implies that we can obtain a $\partial$-compressing disk of $\mathcal{C}_{12}$ such that its boundary is disjoint from $\partial E_{11}$ and that it cuts off a 3 -ball with two mutually trivial arcs from $\mathcal{C}_{12}$. Thus we suppose that $E_{12}$ cuts off a 3 -ball with two mutually trivial arcs from $\mathcal{C}_{12}$. Then $\mathcal{F}_{1}$ is a 2 -sphere intersecting $K$ in two points and hence we have the conclusion (3) of Theorem 7.7 by extraction operation.

Theorem 7.8 Let $K$ be the connected sum of non-trivial knots of $n$-string prime for all $n$ and $\left(\mathcal{C}_{1}, \mathcal{C}_{2} ; \mathcal{S}\right)$ a $(2,2)$-bridge splitting of $\left(S^{3}, K\right)$. Suppose that there are compressing disks $D_{1}$ and $D_{2}$ of $\mathcal{C}_{1}$ and $\mathcal{C}_{2}$ respectively such that $\partial D_{1} \cap \partial D_{2}=\varnothing, D_{1}$ is non-separating, and that $D_{2}$ cuts off a 3-ball with a trivial arc from $\mathcal{C}_{2}$. Suppose also that a torus obtained by the weak reduction with respect to $\left(D_{1}, D_{2}\right)$ is incompressible in $\left(S^{3}, K\right)$. Then one of the following holds.

(1) $\mathcal{S}$ is meridionally stabilized.

(2) $K$ contains a non-trivial 2-bridge knot as a connected summand.

(3) $K=K_{1} \# K_{2}$ such that $K_{1}$ admits a $(1,1)$-bridge position and $K_{2}$ admits a $(1,2)$-bridge position.

Proof As in Figure 11, we obtain $\mathcal{M}_{i}=\mathcal{C}_{i 1} \cup \mathcal{C}_{i 2}, \mathcal{S}_{i}=\mathcal{C}_{i 1} \cap \mathcal{C}_{i 2}$ for each $i=1,2$ and $\mathcal{F}=\partial_{-} \mathcal{C}_{12}=\partial_{-} \mathcal{C}_{21}$ by the weak reduction with respect to $\left(D_{1}, D_{2}\right)$. Recall 
that $\mathcal{F}=(F, F \cap K)$ is a torus of which we suppose incompressibility. It follows from Proposition 7.3 that there is a decomposing sphere of $K$ disjoint from $F$. If a decomposing sphere is contained in $\mathcal{M}_{1}$, then we have a contradiction by the same argument as in the proof of Theorem 7.4. Hence we assume that a decomposing sphere is contained in $\mathcal{M}_{2}$. By an argument similar to the proof of the claim in the proof of Theorem 7.4, we also see that $\mathcal{S}_{2}$ is strongly irreducible. Hence the $\mathrm{cH}$-splitting $\left(\mathcal{C}_{21}, \mathcal{C}_{22} ; \mathcal{S}_{2}\right)$ satisfies one of the conclusions of Corollary 3.4. Moreover, we see that the conclusion (1) of Corollary 3.4 does not hold because $\mathcal{S}_{2}$ is strongly irreducible. Hence we have only the conclusion (2) of Corollary 3.4.

Case 1 There are a compressing disk $E_{21}$ of $\mathcal{C}_{21}$ and a cut disk $E_{22}$ of $\mathcal{C}_{22}$ such that $\partial E_{21} \cap \partial E_{22}=\varnothing$.

If $E_{22}$ is separating in $C_{22}$, then $\mathcal{S}_{2}$ is weakly reducible, a contradiction. Hence $E_{22}$ is a non-separating cut disk of $\mathcal{C}_{22}$. This implies that $\mathcal{S}$ is meridionally stabilized or that there is a non-separating compressing disk of $\mathcal{C}_{21}$ such that its boundary is disjoint from $\partial D_{2} \cup \partial E_{22}$. If the latter occurs, then we have the conclusion (2) or (3) of Theorem 7.8 by Lemma 7.9 which we prove below.

Case 2 There are a separating cut disk $E_{21}$ of $\mathcal{C}_{21}$ and a compressing disk $E_{22}$ of $\mathcal{C}_{22}$ such that $\partial E_{21} \cap \partial E_{22}=\varnothing$.

Then $E_{21}$ cuts $\mathcal{C}_{21}$ into (\{a solid torus $\} \times[0,1]$, two vertical arcs) and (a solid torus $V$, a trivial arc). We may assume that $E_{22}$ is a non-separating compressing disk of $\mathcal{C}_{22}$. Since $\mathcal{F}$ is incompressible in $\left(S^{3}, K\right)$, we see that $\partial E_{22}$ is contained in $\partial V$. This implies that we have the conclusion (3) of Theorem 7.8 by extraction operation (see Figure 14).

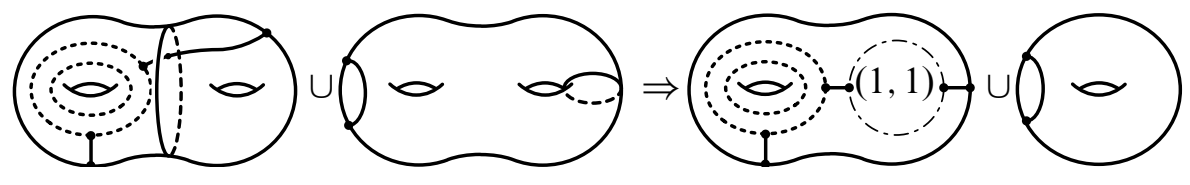

Figure 14: Removing $\eta\left(E_{22}, C_{22}\right)$ from $C_{22}$ and attaching it to $C_{21}$

Case 3 There are a non-separating cut disk $E_{21}$ of $\mathcal{C}_{21}$ and a compressing disk $E_{22}$ of $\mathcal{C}_{22}$ such that $\partial E_{21} \cap \partial E_{22}=\varnothing$. 
It follow from Lemma 3.3 that there is a decomposing sphere $\Sigma$ of $K_{2}=K \cap M_{2}$ such that each component of $\Sigma \cap S_{2}$ is essential in $\mathcal{S}_{2}$, and the components of $\Sigma$ cut along $\Sigma \cap S_{2}$ consist of two disks $\Delta$ and $\Delta^{\prime}$ with $\left|\Delta \cap K_{2}\right|=\left|\Delta^{\prime} \cap K_{2}\right|=1$ and possibly annuli disjoint from $K_{2}$. We take $\Sigma$ so that $\left|\Sigma \cap S_{2}\right|$ is minimal among all such decomposing spheres. If $\Delta$ or $\Delta^{\prime}$ is contained in $C_{22}$, then we have the conclusion (1) of Corollary 3.4 or the condition of Case 1 by an argument in the proof of Corollary 3.4. Hence we assume that both $\Delta$ and $\Delta^{\prime}$ are contained in $C_{21}$. Moreover, if $\Delta$ or $\Delta^{\prime}$ is separating in $C_{21}$, then we have the condition of Case 2. Therefore we also assume that each of $\Delta$ and $\Delta^{\prime}$ is non-separating in $C_{21}$. Then we have either (i) $\Delta$ and $\Delta^{\prime}$ are mutually parallel in $\mathcal{C}_{21}$, or (ii) $\Delta$ and $\Delta^{\prime}$ are not mutually parallel in $\mathcal{C}_{21}$ (see Figure 15).

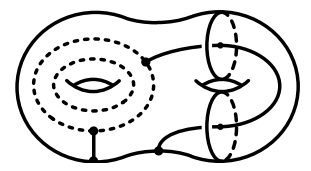

(i)

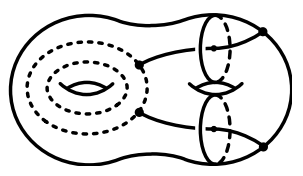

(ii)

Figure 15: Possible positions of $\Delta \cup \Delta^{\prime}$ in $\mathcal{C}_{21}$

We first suppose that $\Delta$ and $\Delta^{\prime}$ satisfy the condition (i). If $\Sigma \cap C_{21}$ contains no annulus component, then $\Sigma \cap C_{22}$ consists of an annulus $A_{22}$. We notice that $A_{22}$ is obtained by joining a compressing disk, which cuts $C_{22}$ into two solid tori, to itself with a band. Hence $A_{22}$ is $\partial$-parallel in $\mathcal{C}_{22}$, because otherwise $M_{2}$ contains a lens space as a connected summand. This implies that $\Sigma$ is isotoped to be contained in $C_{21}$, a contradiction. Therefore $\Sigma \cap C_{21}$ contains an annulus component. We notice that such an annulus component is obtained by joining a non-separating compressing disk to itself with a band. Let $A_{21}$ be the annulus component of $\Sigma \cap C_{21}$ such that $A_{21} \cup \Delta$ or $A_{21} \cup \Delta^{\prime}$, say the former, cuts off a solid torus $V$ with a trivial arc from $\mathcal{C}_{21}$ and that the interior of $V$ is disjoint from $\Sigma$ (see Figure 16). Since $\Sigma \cap C_{22}$ also contains an annulus component, we can obtain a compressing disk $D_{22}$ of $\mathcal{C}_{22}$ by an appropriate $\partial$-compression for a component of $\Sigma \cap C_{22}$. If $V$ is not affected by the $\partial$-compression, then this implies that $\mathcal{S}_{2}$ is weakly reducible, a contradiction. Hence after the $\partial$-compression, $A_{21}$ is joined to $\Delta$ with a band. Let $V^{\prime}$ be the solid torus obtained by cutting $C_{21}$ along $A_{21}$ joined to $\Delta$ with a band. We notice that $V^{\prime}$ is a submanifold of $V$. Attaching $\eta\left(D_{22} ; C_{22}\right)$ to $V^{\prime}$, we obtain a 3-ball $B$ with a single arc. If the arc is trivial in $B$, then we can isotope $\Sigma$ to delete $A_{21}$ and $\Delta$ as components of $\Sigma \cap C_{21}$, contradicting the minimality of $\left|\Sigma \cap S_{2}\right|$. Hence the arc contained in $B$ is non-trivial and therefore $K$ contains a non-trivial 2-bridge knot as a connected summand. Thus we have the conclusion (2) of Theorem 7.8. 


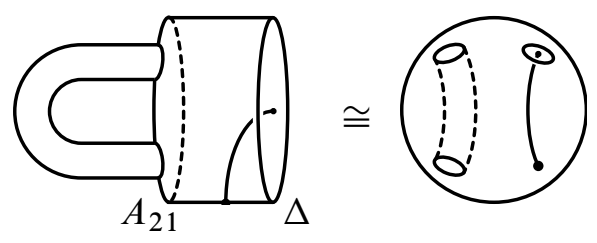

Figure 16: $A_{21} \cup \Delta$ cuts off a solid torus with a trivial arc from $V$

We next suppose that $\Delta$ and $\Delta^{\prime}$ satisfy the condition (ii). Then $\Sigma \cap C_{21}$ contains no annulus component and hence $\Sigma \cap C_{22}$ consists of an annulus $A_{22}^{\prime}$. We notice that $\Delta \cup \Delta^{\prime}$ cuts off a 3-ball with two trivial arcs from $\mathcal{C}_{21}$ and that $A_{22}^{\prime}$ is obtained by joining a separating compressing disk $D_{22}$ of $\mathcal{C}_{22}$ to itself with a band. If $D_{22}$ separates $C_{22}$ into two solid tori, then we see that $\mathcal{S}_{2}$ is weakly reducible, a contradiction. Hence $D_{22}$ separates $\mathcal{C}_{22}$ into a genus two handlebody and a 3 -ball with a trivial arc. This implies that $A_{22}^{\prime}$ cuts off a solid torus with a trivial arc as illustrated at the right side of Figure 16. Therefore $K$ contains a non-trivial 2-bridge knot as a connected summand and hence we have the conclusion (2) of Theorem 7.8.

This completes the proof of Theorem 7.8.

Lemma 7.9 Let $K$ be a knot in $S^{3}$ and $\left(\mathcal{C}_{1}, \mathcal{C}_{2} ; \mathcal{S}\right)$ a $(2,2)$-bridge splitting of $\left(S^{3}, K\right)$. Suppose that there are compressing disks $D_{1}$ and $D_{2}$ of $\mathcal{C}_{1}$ and $\mathcal{C}_{2}$ respectively and a non-separating cut disk $E_{2}$ of $\mathcal{C}_{2}$ such that $\partial D_{1} \cap \partial\left(D_{2} \cup E_{2}\right)=\varnothing, D_{1}$ is non-separating, $D_{2}$ cuts off a 3 -ball with a trivial arc from $\mathcal{C}_{2}$ and $E_{2}$ is disjoint from $D_{2}$. Then one of the following holds.

(1) $\mathcal{S}$ is meridionally stabilized.

(2) $K=K_{1} \# K_{2}$ such that $K_{1}$ admits a $(0,2)$-bridge position and $K_{2}$ admits a $(2,1)$-bridge position.

(3) $K=K_{1} \# K_{2}$ such that $K_{1}$ admits a $(1,1)$-bridge position and $K_{2}$ admits a $(1,2)$-bridge position.

(4) There is a c-weak reduction yielding a 2-sphere which intersects $K$ in four points and is incompressible in $\left(S^{3}, K\right)$.

Proof By the c-weak reduction with respect to $\left(D_{1}, D_{2} \cup E_{2}\right)$, we obtain $\mathcal{M}_{i}=$ $\mathcal{C}_{i 1} \cup \mathcal{C}_{i 2}, \mathcal{S}_{i}=\mathcal{C}_{i 1} \cap \mathcal{C}_{i 2}$ for each $i=1,2$ and $\mathcal{F}=\partial_{-} \mathcal{C}_{12}=\partial_{-} \mathcal{C}_{21}$. We notice that $\mathcal{F}$ is a 2 -sphere intersecting $K$ in four points (see Figure 17). If both $\mathcal{M}_{1}$ and $\mathcal{M}_{2}$ are $\partial$-irreducible, then we have the conclusion (4) of Lemma 7.9. Hence we assume that $\mathcal{M}_{1}$ or $\mathcal{M}_{2}$ is $\partial$-reducible. 

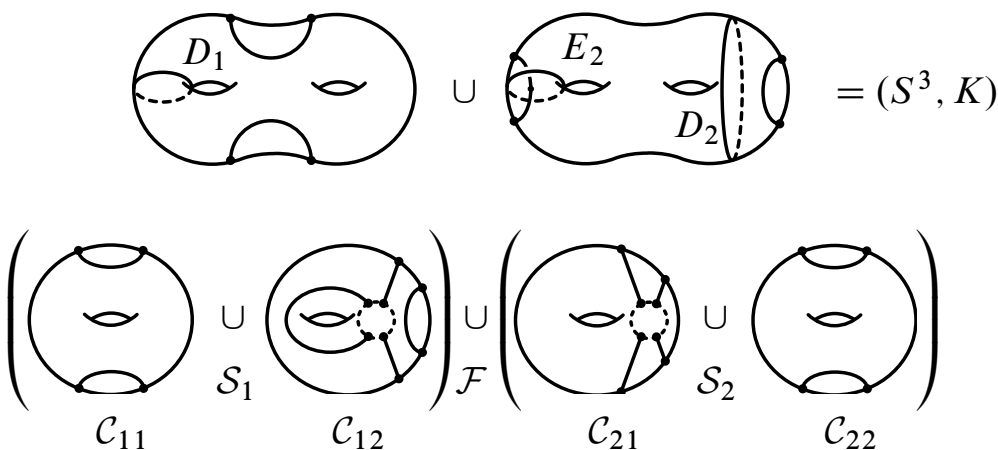

Figure 17: The c-weak reduction with respect to $\left(D_{1}, D_{2} \cup E_{2}\right)$

Suppose that $\mathcal{M}_{1}$ is $\partial$-reducible. Then there is a $\partial$-reducing disk $\bar{D}_{1}$ with $\left|\bar{D}_{1} \cap S_{1}\right|=$ 1 by Lemma 2.10, and there is a compressing disk $D_{12}$ of $\mathcal{C}_{12}$ with $D_{12} \cap \bar{D}_{1}=\varnothing$ by Corollary 2.11. It follows from Lemma 2.4 that $D_{12}$ is isotopic to $\widehat{D}_{2}$ in $\mathcal{C}_{12}$, where $\widehat{D}_{2}$ is a compressing disk of $\mathcal{C}_{12}$ which is obtained by extending $D_{2}$ naturally. Hence we see that $\bar{D}_{1} \cap \widehat{D}_{2}=\varnothing$. The disk $\bar{D}_{1}$ can be regarded as a compressing disk of $\mathcal{C}_{1}$ which is disjoint from $D_{1}$ and is not parallel to $D_{1}$. We notice that $\partial\left(D_{1} \cup \bar{D}_{1}\right) \cap \partial D_{2}=\varnothing$. Hence we have the conclusion (1) or (2) of Lemma 7.9 (see Figure 6).

Suppose that $\mathcal{M}_{2}$ is $\partial$-reducible. Then there is a $\partial$-reducing disk $\bar{D}_{2}$ with $\left|\bar{D}_{2} \cap S_{2}\right|=$ 1 by Lemma 2.10, and there is a compressing disk $D_{21}$ of $\mathcal{C}_{21}$ with $D_{21} \cap \bar{D}_{2}=\varnothing$ by Corollary 2.11. Since we may assume that $D_{21}$ is non-separating in $C_{21}$, it follows from Lemma 2.4 that $D_{21}$ is isotopic to $D_{1}$ in $\mathcal{C}_{21}$. Hence we see that $D_{1} \cap \bar{D}_{2}=\varnothing$. The disk $\bar{D}_{2}$ can be regarded as a compressing disk of $\mathcal{C}_{2}$ which is disjoint from $D_{2} \cup E_{2}$ and is parallel neither to $D_{2}$ nor to $E_{2}$. We notice that $\partial D_{1} \cap \partial\left(D_{2} \cup E_{2} \cup \bar{D}_{2}\right)=\varnothing$. Hence we have the conclusion (3) of Lemma 7.9.

Proof of Theorem 1.4 The proof for (2) of Theorem 1.4 is quite similar to that for (1). Hence we give a proof only for (1) of Theorem 1.4. Let $K_{i}(i=1,2,3)$ be knots in $S^{3}$ with $K_{i} \in \mathcal{K}_{2}^{1}$. We notice that $\operatorname{hg}\left(K_{1} \# K_{2}\right)=3$ and $K_{1} \# K_{2} \notin \mathcal{K}_{3}^{3}$ (see Observation 1.3). It follows from Observation 1.3, Theorems 4.1 and 4.2 that $K_{1} \# K_{2}$ cannot admit a $(1,2)$-bridge position, that is, $K_{1} \# K_{2} \notin \mathcal{K}_{3}^{2}$. On the other hand, we see that $K_{1} \# K_{2}$ admits a $(2,1)$-bridge position by Proposition 2.14 . Hence we see that $K_{1} \# K_{2} \in \mathcal{K}_{3}^{1}$.

We now consider meridional destabilizing number of $K_{1} \# K_{2} \# K_{3}$. We notice that $\operatorname{hg}\left(K_{1} \# K_{2} \# K_{3}\right)=4$ and $K_{1} \# K_{2} \# K_{3} \notin \mathcal{K}_{4}^{4}$ (see Observation 1.3). Suppose first that $K_{1} \# K_{2} \# K_{3}$ admits a $(1,3)$-bridge position. Since each $K_{i}$ is $n$-string prime for all $n$ (see Gordon and Reid [3, Corollary 1.2]), we have the conclusion (2) or (4) of 
Theorem 5.2 by Section 5. If the conclusion (2) of Theorem 5.2 holds, then $K_{i} \# K_{j}$, say $(i, j)=(1,2)$, must admit a $(0,3)$-bridge position because $K_{3} \in \mathcal{K}_{2}^{1}$. This, however, implies that $K_{1}$ admits a $(0,2)$-bridge position (see Observation 1.3), contradicting $K_{1} \in \mathcal{K}_{2}^{1}$. It follows from Theorem 7.4 that the conclusion (4) of Theorem 5.2 is impossible, because each $K_{i}$ is $n$-string prime for all $n$. Thus we see that $K_{1} \# K_{2} \# K_{3}$ does not admit a $(1,3)$-bridge position, that is, $K_{1} \# K_{2} \# K_{3} \notin \mathcal{K}_{4}^{3}$. Suppose next that $K_{1} \# K_{2} \# K_{3}$ admits a $(2,2)$-bridge position. Then by Section 6 , we have the conclusion (3) of Theorem 6.1, the conclusion (2), (4) or (5) of Theorem 6.2. If the conclusion (2) of Theorem 6.2 holds, then $K_{i} \# K_{j}$, say $(i, j)=(1,2)$, must admit a $(1,2)$-bridge position, contradicting $K_{1}, K_{2} \in \mathcal{K}_{2}^{1}$ by Section 4 . The conclusion (3) of Theorem 6.1 is impossible by Theorem 7.5. If the conclusion (4) or (5) of Theorem 6.2 holds, then we see that $K_{1} \# K_{2} \# K_{3}$ contains a non-trivial 2-bridge knot as a connected summand by Theorems 7.6-7.8, a contradiction. Hence we see that $K_{1} \# K_{2} \# K_{3}$ does not admit a $(2,2)-$ bridge position, that is, $K_{1} \# K_{2} \# K_{3} \notin \mathcal{K}_{4}^{2}$. On the other hand, we see that $K_{1} \# K_{2} \# K_{3}$ admits a $(3,1)$-bridge position by Proposition 2.14 . Therefore we see that $K_{1} \# K_{2} \# K_{3} \in \mathcal{K}_{4}^{1}$.

\section{Acknowledgements}

The author was supported by JSPS Postdoctoral Fellowships for Research Abroad. This work was carried out while the author was visiting at University of California, Santa Barbara. He would like to express his thanks to the department for its hospitality. He would also like to thank Martin Scharlemann for helpful conversations. He is also grateful to Yo'av Rieck for useful comments, particularly for pointing out Remark 1.6.

\section{References}

[1] A J Casson, C M Gordon, Reducing Heegaard splittings, Topology Appl. 27 (1987) 275-283 MR918537

[2] H Doll, A generalized bridge number for links in 3-manifolds, Math. Ann. 294 (1992) 701-717 MR1190452

[3] C M Gordon, A W Reid, Tangle decompositions of tunnel number one knots and links, J. Knot Theory Ramifications 4 (1995) 389-409 MR1347361

[4] C Hayashi, K Shimokawa, Thin position of a pair (3-manifold, 1-submanifold), Pacific J. Math. 197 (2001) 301-324 MR1815259

[5] J Johnson, A Thompson, On tunnel number one knots which are not $(1, n)$ arXiv: math.GT/0606226

[6] T Kobayashi, Y Rieck, Heegaard genus of the connected sum of m-small knots, Comm. Anal. Geom. 14 (2006) 1037-1077 MR2287154 
[7] T Kobayashi, Y Rieck, Knot exteriors with additive Heegaard genus and Morimoto's conjecture, Algebr. Geom. Topol. 8 (2008) 953-969 MR2443104

[8] T Kobayashi, Y Rieck, Knots with $g(E(K))=2$ and $g(E(K \# K \# K))=6$ and Morimoto's conjecture, Topology Appl. 156 (2009) 1114-1117 MR2493371

[9] T Kobayashi, T Saito, Destabilizing Heegaard splittings of knot exteriors, Topology Appl. 157 (2010) 202-212 MR2556098

[10] Y N Minsky, Y Moriah, S Schleimer, High distance knots, Algebr. Geom. Topol. 7 (2007) 1471-1483 MR2366166

[11] K Morimoto, On the additivity of tunnel number of knots, Topology Appl. 53 (1993) 37-66 MR1243869

[12] K Morimoto, Tunnel number, connected sum and meridional essential surfaces, Topology 39 (2000) 469-485 MR1746903

[13] K Morimoto, Characterization of composite knots with 1-bridge genus two, J. Knot Theory Ramifications 10 (2001) 823-840 MR1840270

[14] K Morimoto, M Sakuma, Y Yokota, Examples of tunnel number one knots which have the property “ $1+1=3$ ”, Math. Proc. Cambridge Philos. Soc. 119 (1996) 113-118 MR1356163

[15] M Scharlemann, J Schultens, The tunnel number of the sum of $n$ knots is at least $n$, Topology 38 (1999) 265-270 MR1660345

[16] H Schubert, Über eine numerische Knoteninvariante, Math. Z. 61 (1954) 245-288 MR0072483

[17] J Schultens, Additivity of tunnel number for small knots, Comment. Math. Helv. 75 (2000) 353-367 MR1793793

[18] M Tomova, Thin position for knots in a 3-manifold, J. Lond. Math. Soc. (2) 80 (2009) 85-98 MR2520379

Department of Mathematics, University of California at Santa Barbara Santa Barbara CA 93106, USA

tsaito@math.ucsb.edu

Received: 14 December 2010 Revised: 9 February 2011 